\title{
Substrate-Controlled Michael Additions of Titanium Enolates from Chiral $\alpha$-Benzyloxy Ketones to Conjugated Nitroalkenes
}

\author{
Alejandro Gómez-Palomino, ${ }^{[a]}$ Adrián Barrio, ${ }^{[a]}$ Pedro García-Lorente,${ }^{[a]}$ Pedro Romea, ${ }^{*[a]}$ Fèlix Urpít ${ }^{*[a]}$ \\ and Mercè Font-Bardia ${ }^{[b]}$
}

\begin{abstract}
Lewis acid-mediated substrate-controlled reactions of the titanium(IV) enolates of chiral $\alpha$-benzyloxy ketones with conjugated nitroalkenes give the 2,4-anti-4,5-syn Michael adducts in good yields and diastereomeric ratios. The supplementary Lewis acid plays a key role in the outcome of these transformations, probably as a consequence of the formation of bimetallic enolates that increase the reactivity of the enolate and direct the approach of the nitroalkene. Importantly, the most appropriate Lewis acid depends on the electrophilic partner: $\mathrm{TiCl}_{4}$ is the most suitable Lewis acid for $\beta$-aryl nitroalkenes while the best results for $\beta$-alkyl nitroalkenes are obtained with $\mathrm{SnCl}_{4}$. Finally, the nitro group of the resultant compounds can be converted into the corresponding amino, oxime, and nitrile groups under mild conditions, which permits the synthesis of a variety of enantiomerically pure derivatives with excellent yields.
\end{abstract}

\section{Introduction}

The venerable Michael reaction, which refers to additions of stable carbon nucleophiles to conjugated olefins bearing an electron-withdrawing group, is beyond doubt one of the most powerful carbon-carbon bond forming reactions. ${ }^{[1]}$ Indeed, the wide range of structural motifs it gives access to and the possibility of installing up to three new stereocenters sustain a great and lingering interest in the Michael reaction within synthetic chemistry. Thus, tremendous effort has been dedicated to developing asymmetric procedures that permit control of the configuration of as many stereocenters as possible, ${ }^{[2,3]}$ and a variety of recently reported enantioselective and catalytic Michael additions have already been employed in the synthesis of a plethora of natural products. ${ }^{[4,5]}$ Unfortunately, and despite such achievements, most of these methods hinge on the use of metal enolates from 1,3-dicarbonyl compounds, ${ }^{[6]}$ and other activated substrates, ${ }^{[7]}$ as the nucleophilic partner, which restricts their scope. ${ }^{[8]}$ Meanwhile, more classic stoichiometric and chiral auxiliary-based approaches take advantage of the high reactivity of lithium and titanium(IV)

[a] Departament de Química Inorgànica i Orgànica, Secció de Química Orgànic, and Institut de Biomedicina (IBUB)

Universitat de Barcelona

Carrer Martí i Franqués 1-11, 08028, Barcelona, Catalonia, Spain

E-mail: pedro.romea@ub.edu; felix.urpi@ub.edu

http://www.qo.ub.edu/grups/SSNP/en/qui_som_presentacio.html

[b] Unitat de Difracció de R-X. CCiTUB

Universitat de Barcelona

Carrer Solé i Sabarís 1-3, 08028 Barcelona, Catalonia, Spain

Supporting information and ORCID(s) from the author(s) for this article are available on the WWW under.((Please delete this text if not appropriate)) enolates to tackle more challenging additions. ${ }^{[9]}$ Surprisingly parallel substrate-controlled Michael reactions from chiral ketones that might achieve similar levels of stereocontrol are almost unknown, and only a few examples have been reported to date. ${ }^{10,11}$

Therefore, considering that titanium(IV) enolates from chiral $\alpha$ benzyloxy ketones take part in highly stereocontrolled aldol reactions, ${ }^{[12]}$ we envisaged that they might also participate in diastereoselective Michael reactions. We were pleased to discover that enones are excellent acceptors and afforded the corresponding 1,5-dicarbonyl compounds in high yields and diastereomeric ratios (see eq 1 in Scheme 1). ${ }^{[13]}$ Then, bearing in mind that $\alpha, \beta$-unsaturated nitro derivatives are more active Michael acceptors than enones, and also the various transformations available for the nitro group, ${ }^{[14]}$ we hypothesized that substrate-controlled Michael additions of the aforementioned chiral ketones to conjugated nitroalkenes might afford the corresponding adducts, which could in turn be easily converted into a range of enantiomerically pure intermediates. Herein, we report that the Lewis acid-mediated addition of titanium(IV) enolates from chiral $\alpha$-benzyloxy ketones to a wide range of nitroalkenes affords 2,4-anti-4,5-syn adducts with good yields and diastereoselectivities (see eq 2 in Scheme 1).

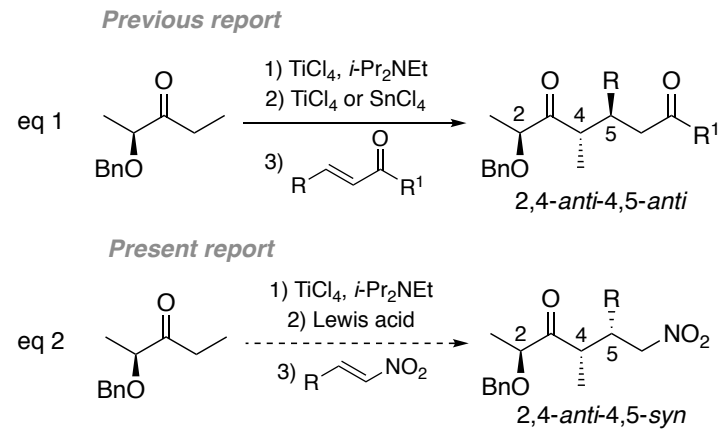

Scheme 1. Substrate-controlled Michael additions from $\alpha$-benzyloxy ketones.

\section{Results and Discussion}

Taking advantage of our experience with substrate-controlled Michael additions to enones, ${ }^{[13]}$ we initially assessed the Lewis acid-mediated reaction of lactate-derived ketone $1^{[15]}$ to $\beta$ nitrostyrene (a). Surprisingly, and in spite of the strong character of $\beta$-nitrostyrene (a) as a Michael acceptor, the simple reaction with the titanium(IV) enolate from 1 did not produce any Michael adduct, and the starting materials were recovered unchanged (entry 1 in Table 1). In contrast, full conversion was achieved by 
Table 1. Lewis acid-mediated Michael additions of titanium(IV) enolates from 1 to $\beta$-nitrostyrene.

\begin{tabular}{|c|c|c|c|c|c|c|}
\hline & $\overbrace{\mathrm{BnO}}^{\mathrm{O}}$ & $\frac{i-\mathrm{Pr}_{2} \mathrm{NEt}}{2,-78^{\circ} \mathrm{C}}$ & $\gamma^{\ominus}$ & $\begin{array}{l}\text { uiv Lewis acid } \\
\text { ench, } t_{\text {quench }}\end{array}$ & 5 & \\
\hline Entry & Lewis acid & $t_{\text {reac }}[\mathrm{h}]$ & Quench & $t_{\text {quench }}[\mathrm{min}]$ & $d r^{[a]}$ & Yield $2 \mathrm{a}[\%]^{[\mathrm{b}]}$ \\
\hline 1 & - & 1.5 & $\mathrm{NH}_{4} \mathrm{Cl}$ & 10 & - & - \\
\hline 2 & $\mathrm{TiCl}_{4}$ & 1.5 & $\mathrm{NH}_{4} \mathrm{Cl}$ & 10 & nd & 41 \\
\hline 3 & $\mathrm{TiCl}_{4}$ & 1.5 & $\mathrm{HCl}$ & 10 & nd & 47 \\
\hline 4 & $\mathrm{TiCl}_{4}$ & 1.5 & $\mathrm{NH}_{4} \mathrm{~F}$ & 90 & $87: 13$ & 79 \\
\hline 5 & $\mathrm{TiCl}_{4}$ & 1 & $\mathrm{NH}_{4} \mathrm{~F}$ & 30 & $87: 13$ & 80 \\
\hline 6 & $\mathrm{AlEt}_{2} \mathrm{Cl}$ & 1.5 & $\mathrm{NH}_{4} \mathrm{~F}$ & 30 & $75: 25$ & $(60)^{[c]}$ \\
\hline 7 & $\mathrm{SnCl}_{4}$ & 1.5 & $\mathrm{NH}_{4} \mathrm{~F}$ & 30 & $50: 50$ & $(82)^{[c]}$ \\
\hline
\end{tabular}

[a] Diastereomeric ratio established by ${ }^{1} \mathrm{H}$ NMR $(400 \mathrm{MHz})$. [b] Isolated yield of 2a. [c] Overall yield of all the diastereomers.

adding a second equivalent of $\mathrm{TiCl}_{4}$ to the enolate and quenching the reaction mixture with saturated $\mathrm{NH}_{4} \mathrm{Cl}$; however, unexpectedly, the diastereomerically pure adduct $\mathbf{2 a}$ was isolated with only a moderate yield after chromatographic purification (entry 2 in Table 1). The use of a stronger Brønsted acid to quench the reaction failed to increase the yield (entry 3 in Table 1). Careful analysis of the literature revealed that Seebach had faced a similar problem. Indeed, he found that the addition of titanium(IV) enolates from chiral imides to conjugate nitroalkenes gave stable nitronates that were not satisfactorily released using standard acid treatments. ${ }^{[16]}$ Instead, it was necessary to quench the reaction with $\mathrm{NH}_{4} \mathrm{~F}$. By subjecting our reaction to such conditions we obtained an 87:13 diastereomeric mixture, from which adduct $2 \mathrm{a}$ was isolated with an $80 \%$ yield (entries 4 and 5 in Table 1). Finally, other Lewis acids such as $\mathrm{AlEt}_{2} \mathrm{Cl}$ and $\mathrm{SnCl}_{4}$ were also tested, but both the diastereoselectivity and the yield were poorer than those obtained with $\mathrm{TiCl}_{4}$ (compare entries 5-7 in Table 1).

Having established the key role of a second equivalent of $\mathrm{TiCl}_{4}$ and the importance of quenching the reaction mixture with $\mathrm{NH}_{4} \mathrm{~F}$, we then assessed reactions with a variety of $\beta$-aryl nitroalkenes (see Table 2). ${ }^{[17]}$ With the exception of the 4-nitrophenyl derivative $\mathbf{f}$, all the substrates examined reacted smoothly to afford mixtures from which 2,4-anti-4,5-syn 2 diastereomers were isolated in high yields (entries 1-6 in Table 2). Remarkably,

Table 2. $\mathrm{TiCl}_{4}-$ Mediated Michael additions of titanium(IV) enolates from 1 to $\beta$-aryl nitroalkenes.

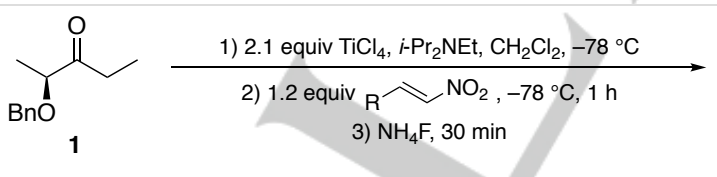

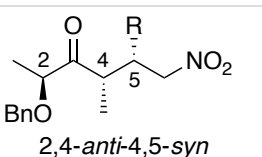

2

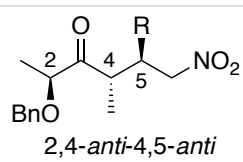

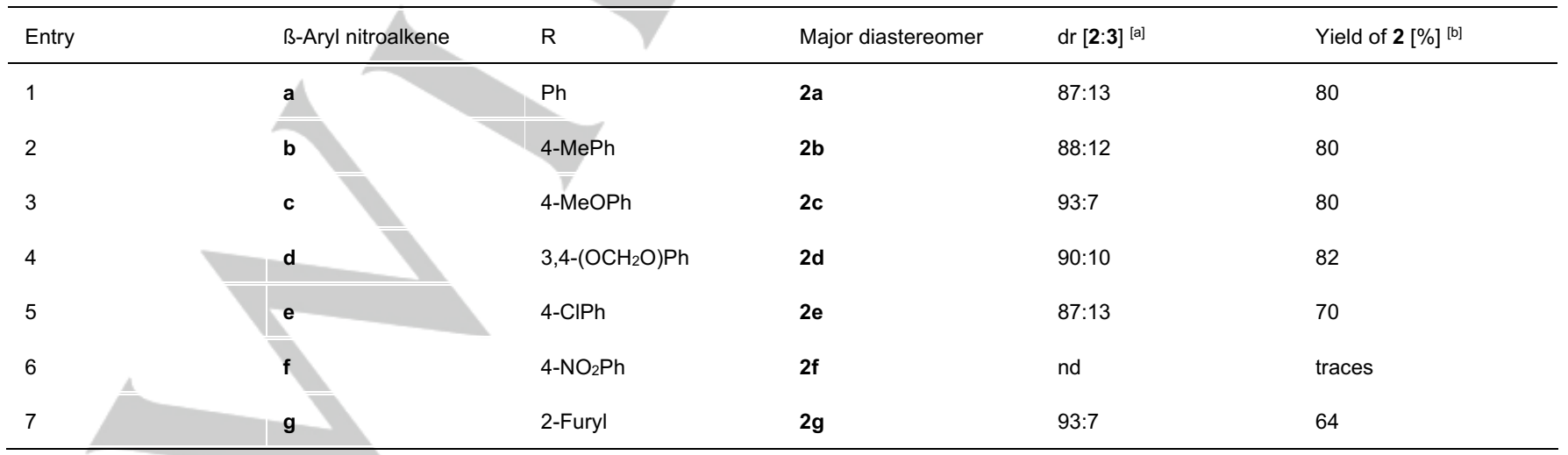

[a] Diastereomeric ratio established by ${ }^{1} \mathrm{H}$ NMR $(400 \mathrm{MHz})$. [b] Isolated yield. 
even the addition of $\mathbf{1}$ to a Lewis acid-sensitive acceptor such as furyl nitroalkene $\mathbf{g}$ proceeded, and led to a good yield (entry 7 in Table 2). Interestingly, the diastereoselectivity of the reaction was observed to be dependent in some way on the electronic character of the aromatic ring: it was slightly higher for those substrates containing electron-donating groups on the aromatic ring, such as c (entry 3 in Table 2).

With the aim of expanding the scope of the process, we next examined parallel conjugate additions to $\beta$-alkyl nitroalkenes. To our surprise, the reaction of 1 with $(E)$-1-nitro-4-phenyl-1-butene (h) gave the expected product $\mathbf{2 h}$ but only with moderate stereocontrol and yield (dr 60:40,60\% overall yield, see entry 1 in Table 3). Then, considering the crucial impact of the second equivalent of Lewis acid on the outcome of these additions, we evaluated the influence of this component on the addition to $\beta$ alkyl nitroalkenes. After careful analysis, we observed that other titanium(IV) as well as zirconium(IV) or aluminum Lewis acids produced similar or even worse results; but $\mathrm{SnCl}_{4}$ provided much better diastereoselectivity with a comparable yield (compare entries 1-6 in Table 3). Indeed, treatment of the titanium(IV) enolate from 1 with one equivalent of $\mathrm{SnCl}_{4}$ before the addition of the nitroalkene $\mathbf{h}$ yielded a mixture of three diastereomers ( $d r$ 84:10:6) from which adduct $\mathbf{2 h}$ was isolated with a $46 \%$ yield (entry 6 in Table 3 ). An increase of the temperature to $-40{ }^{\circ} \mathrm{C}$ improved the yield, but a higher temperature had a negative impact on the yield (compare entries $6-8$ in Table 3). Finally, a longer reaction time also improved the yield (compare entries 6-9 in Table 3 ), so diastereomerically pure $\mathbf{2 h}$ was finally isolated with a $54 \%$ yield after $3 \mathrm{~h}$ at $-78{ }^{\circ} \mathrm{C}$ (entry 9 in Table 3 ). These optimized experimental conditions were further applied to other $\beta$-alkyl nitroalkenes $(\mathbf{i}-\mathbf{m})^{[18]}$ to afford 2,4-anti-4,5-syn adducts $\mathbf{2 i - m}$ with high stereocontrol. Interestingly, the addition proved to be very sensitive to the steric bulk and the presence of potential chelating functional groups on R. Indeed, the addition of the titanium enolate from 1 to $(E)$-3-methyl-1-nitro-1-butene $(\mathbf{k})$ in which an isopropyl group is attached to the electrophilic center gave the 2,4-anti-4,5-syn adduct $\mathbf{2 k}$ with a low diastereoselectivity and yield (entry 12 in Table 3). In turn, the reaction with benzyl-protected 4-hydroxy-1nitro-1-butene (I) gave 2I with an excellent diastereoselectivity but in a low yield (entry 13 in Table 3); whereas the use of a

Table 3. Lewis acid-mediated Michael additions of titanium(IV) enolates from 1 to $\beta$-alkyl nitroalkenes.

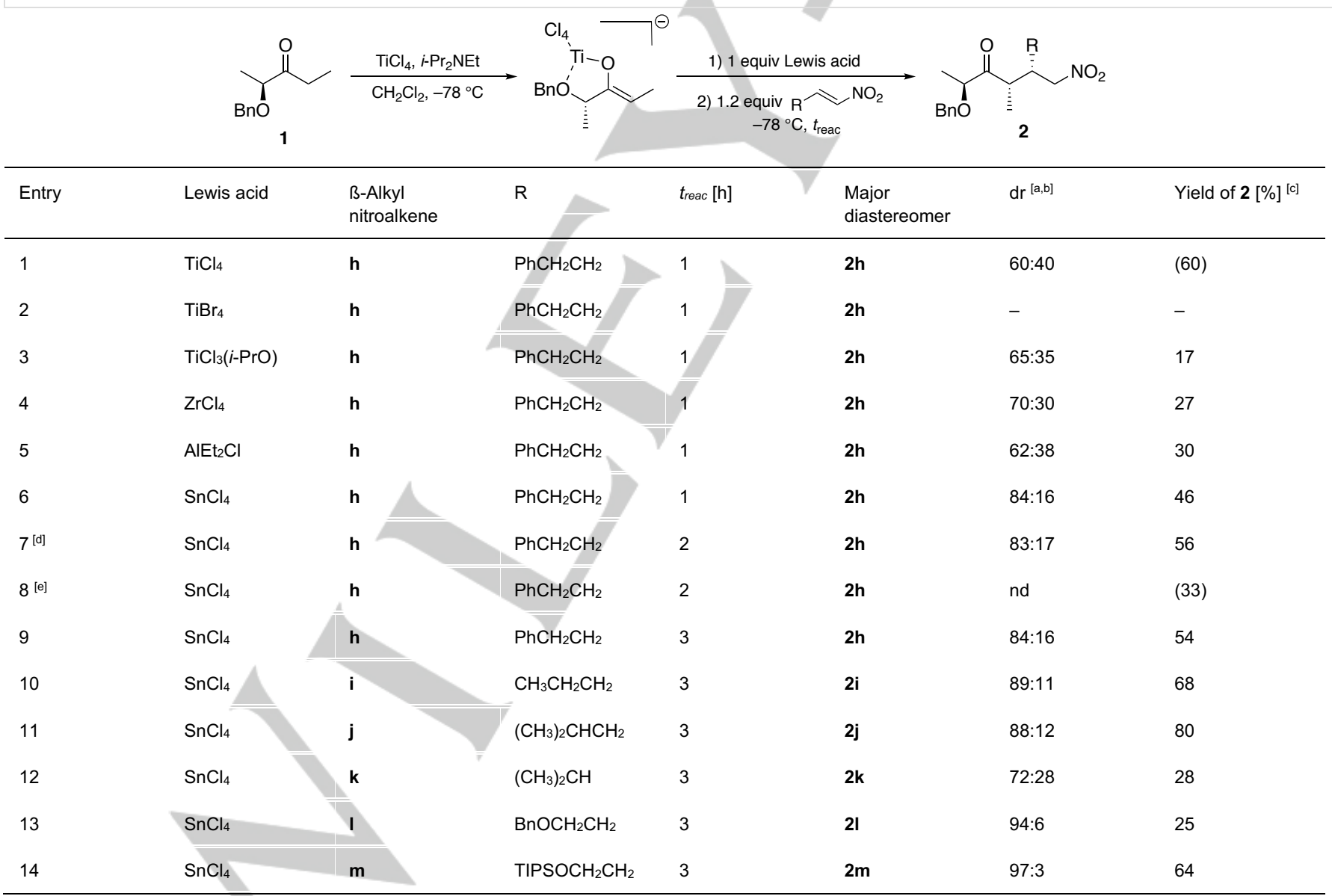

[a] Ratio of major/sum of minor diastereomers. [b] Diastereomeric ratio established by ${ }^{1} \mathrm{H}$ NMR $(400 \mathrm{MHz})$. [c] Isolated yield. [d] The reaction was carried out at $78{ }^{\circ} \mathrm{C}$ for $1 \mathrm{~h}$ and at $-40{ }^{\circ} \mathrm{C}$ for $1 \mathrm{~h}$. [e] The reaction was carried out at $-78{ }^{\circ} \mathrm{C}$ for $1 \mathrm{~h}$ and at $-20{ }^{\circ} \mathrm{C}$ for $1 \mathrm{~h}$. 
bulky and non-chelating TIPS protecting group in $\mathbf{m}$ provided the desired addutc $2 \mathrm{~m}$ as a single diastereomer with a good yield (entry 14 in Table 3).

The configurations of major and minor diastereomer, 2 and $\mathbf{3}$ respectively, were established through X-ray analyses of crystalline adducts $\mathbf{2} \mathbf{g}$ and $\mathbf{3 a}$ shown in Figure 1. ${ }^{[19]}$

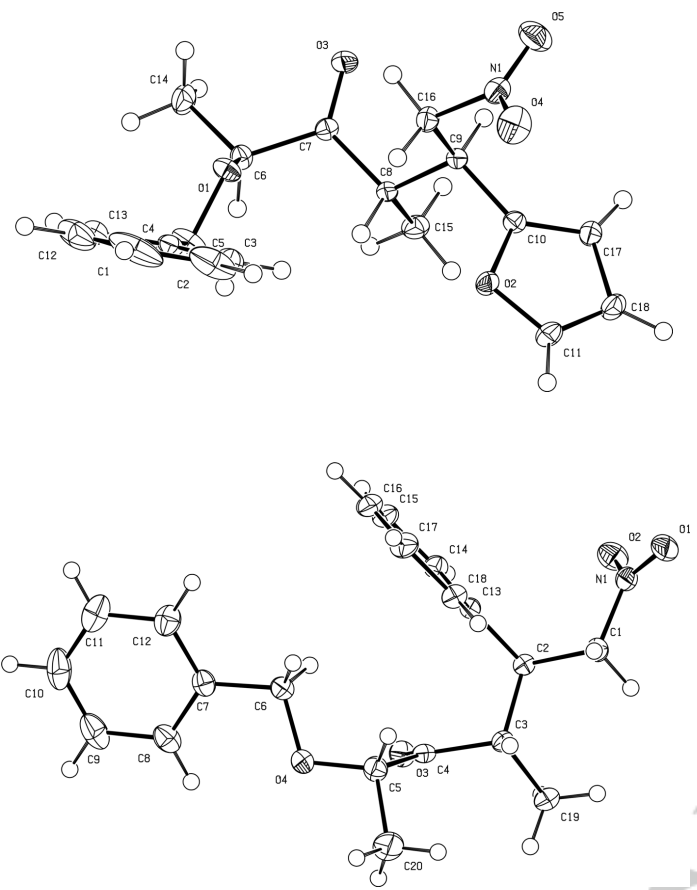

Figure 1. ORTEP $X$-ray structures of compounds $\mathbf{2} \mathbf{g}$ and $\mathbf{3 a}$ [ellipsoid contour probability: $50 \%$ ]

The broad scope of the abovementioned Lewis acid-mediated Michael additions of $\mathbf{1}$ to conjugated nitroalkenes encouraged us to explore similar substrate-controlled reactions with other chiral $\alpha$-hydroxy ketones. Thus, we were pleased to observe that the titanium(IV) enolates from $\alpha$-benzyloxy ketones $\mathbf{4}$ and $\mathbf{5}^{[20]}$ smoothly added to $\beta$-nitrostyrene (a) to afford basically a single diastereomer $\mathbf{6 a - 7 a}$ in yields of up to $72 \%$ (Scheme 2 ).

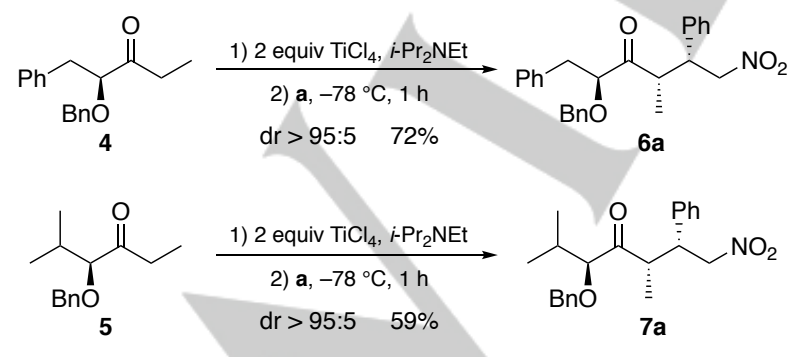

Scheme 2. Substrate-controlled Michael additions from $\alpha$-benzyloxy ketones.

All together, these results prove that chiral $\alpha$-benzyloxy ketones are excellent platforms from which to carry out highly stereoselective Lewis acid-mediated Michael reactions with a wide range of nitroalkenes. As for enones, the supplementary Lewis acid plays a crucial role in these additions. Indeed, $\mathrm{TiCl}_{4}$ and $\mathrm{SnCl}_{4}$ turned out to be the most suitable Lewis acids, depending on the acceptor, $\beta$-aryl or $\beta$-alkyl nitroalkenes respectively. This indicates that the second Lewis acid must interact with the enolate, producing the real nucleophilic species (see Scheme 3), which must also be responsible for the activation of the nitroalkene.

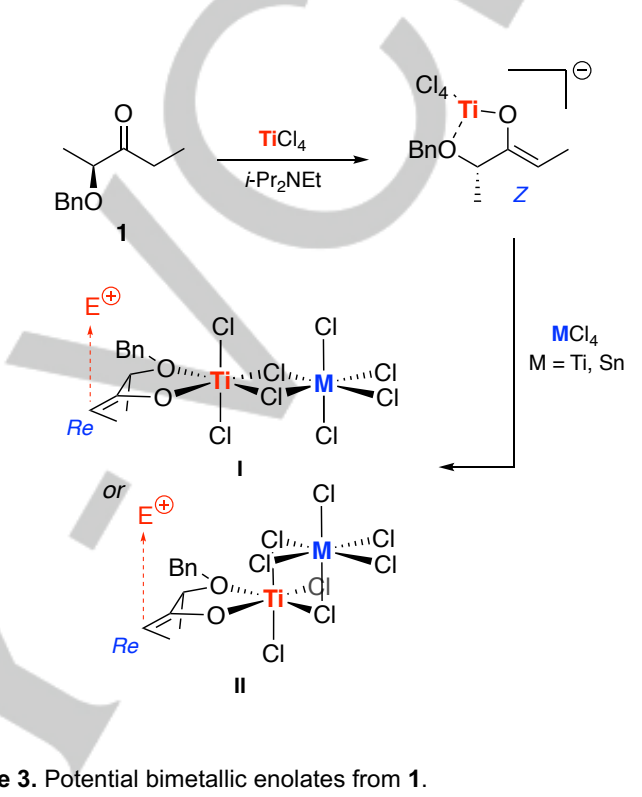

Irrespective of the precise structure of such an intermediate and according to the structure both of the major and minor diastereomers shown in Figure 1, the electrophile would attack the $R e$ face of the enolate, thus controlling the configuration of the $\alpha$-stereocenter. In turn, that of the $\beta$-stereocenter relies on the approach of the electrophile to the $R e$ face of the enolate. Enones produce the relative configuration anti; nitroalkenes the syn configuration (see Scheme 1). The reasons for such a discrepancy may be conformational differences operating on the activation of the double bond. Indeed, the equilibrium for enones is shifted towards the cisoid conformation which avoids $A(1,3)$ interactions, whereas the lack of an $R^{2}$ group in nitroalkenes makes the transoid conformation more accessible. ${ }^{[21]}$ Actually, both conformers of nitroalkenes may be considered as tautomers in which the metal atom is bound to different oxygen atoms of the nitro group. Moreover, the key role played by the additional Lewis acid suggests that the reaction might proceed through a cyclic transition state involving a bimetallic enolate; ${ }^{[22]}$ then, the essentially flat nitroalkene does not contain an $R^{2}$ group that would prevent it from evolving through an eclipsed approach (I in Scheme 4), which looks like an eight-membered cycle. ${ }^{[23]}$ Our working hypothesis precisely predicted such an approach as being responsible for the 4,5-syn diastereomer, whereas the 4,5-anti counterpart may arise from a staggered approach (II in Scheme 4). So, slight differences in the electronic character of nitroalkenes (aryl or alkyl) and the steric bulk of $\mathrm{R}^{1}$ 
can have a dramatic impact on the stereochemical outcome of these additions.

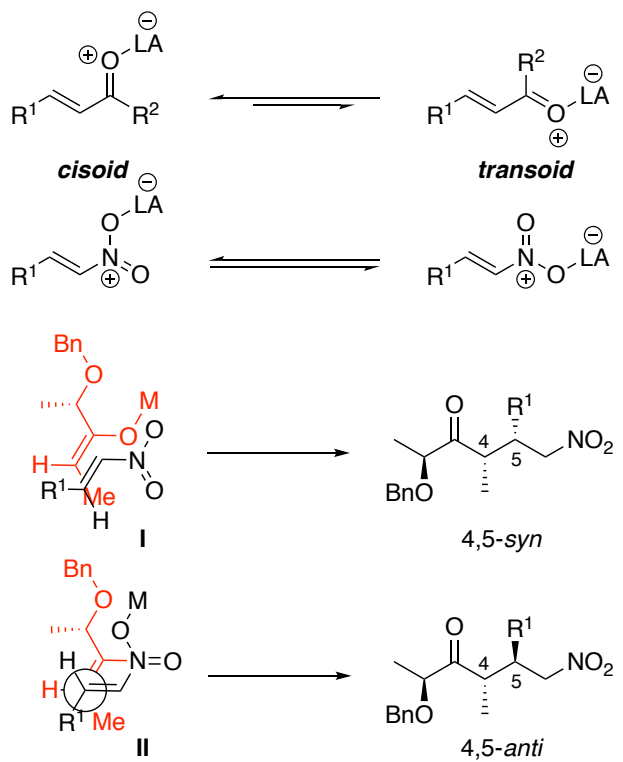

Scheme 4. Mechanistic hypothesis.

Finally, we explored the conversion of the nitro group into other functional groups, to confirm their synthetic potential. Thus, the reduction of the nitro group of adduct $2 \mathrm{a}$ catalyzed by $\mathrm{NiCl}_{2}$ led to enantiomerically pure cyclic imine 8 in $90 \%$ yield (Scheme 5). ${ }^{[24,6 c]}$ Moreover, 2a was easily converted into oxime 9 using a tin-based reducing agent (Scheme 5). ${ }^{[25]}$ Finally, a reductive dehydration of 2 a catalyzed by $\mathrm{Sn}(\mathrm{SPh})_{4}$ allowed us to isolate $\beta$ cyano ketone 10 with an excellent yield (Scheme 5). ${ }^{[26]}$ All these reactions were carried out under mild conditions and the resulting densely functionalized compounds 8-10 were easily isolated in high yields and without any loss of the steric integrity of the starting material.

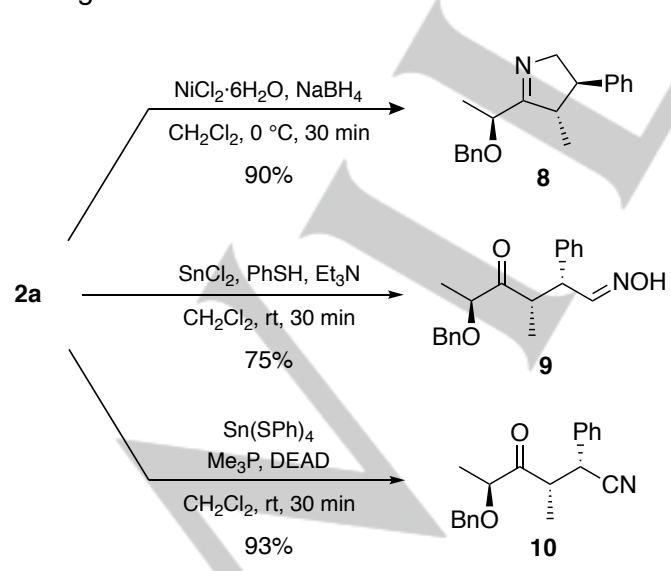

Scheme 5. Further transformations from adduct 2a

\section{Conclusions}

In summary, Lewis acid-mediated substrate-controlled Michael additions of $\alpha$-benzyloxy ketones to a wide range of $\beta$-aryl- as well as $\beta$-alkyl-conjugated nitroalkenes afford the corresponding 2,4-anti-4,5-syn diastereomers with good yields and high stereocontrol. It is probable that a bimetallic enolate arising from the interaction of the titanium(IV) enolate with the supplementary Lewis acid $\left(\mathrm{TiCl}_{4}\right.$ or $\left.\mathrm{SnCl}_{4}\right)$ is responsible for the syn trend observed for these additions. Furthermore, simple transformations of the nitro group offer access to other nitrogenbased functional groups in a straightforward manner.

\section{Experimental Section}

General: Unless otherwise noted, reactions were conducted in ovendried glassware under inert atmosphere of $\mathrm{N}_{2}$ with anhydrous solvents. The solvents and reagents were dried and purified when necessary according to standard procedures. Commercially available reagents were used as received. Analytical thin-layer chromatography (TLC) was carried out on Merck silica gel $60 \mathrm{~F}_{254}$ plates and analyzed by UV (254 $\mathrm{nm}$ ) and stained with phosphomolybdic acid and $p$-anisaldehyde; $R_{f}$ values are approximate. Column chromatographies were carried out under low pressure (flash) conditions and performed on SDS silica gel 60 $(35-70 \mu \mathrm{m})$. Specific rotations $\left([\alpha]_{\mathrm{D}}\right)$ were determined at $20{ }^{\circ} \mathrm{C}$ on a Perkin-Elmer 241 MC polarimeter. IR spectra (Attenuated Total Reflectance, ATR) were recorded on a Nicolet 6700 FT-IR Thermo Scientific spectrometer and only the more representative frequencies $(v)$ are reported in $\mathrm{cm}^{-1} .{ }^{1} \mathrm{H}$ NMR $(400 \mathrm{MHz})$ and ${ }^{13} \mathrm{C} \mathrm{NMR}(100.6 \mathrm{MHz})$ spectra were recorded on a Varian Mercury 400. Chemical shifts $(\delta)$ are quoted in ppm and referenced to internal TMS ( $\delta 0.00$ for ${ }^{1} \mathrm{H}$ NMR) or $\mathrm{CDCl}_{3}$ ( $\delta 77.0$ for ${ }^{13} \mathrm{C} \mathrm{NMR}$ ); coupling constants $(J)$ are quoted in $\mathrm{Hz}$; data are reported as follows: $\mathrm{s}$, singlet; $\mathrm{d}$, doublet; $\mathrm{t}$, triplet; $\mathrm{q}$, quartet; $\mathrm{m}$, multiplet (and their corresponding combinations); where necessary, 2D techniques (NOESY, COSY, HSQC) were also used to assist on structure elucidation. High resolution mass spectra (HRMS) were obtained with an Agilent 1100 spectrometer by the Unitat d'Espectrometria de Masses (CCiTUB), Universitat de Barcelona.

General Experimental Procedure for the $\mathrm{TiCl}_{4}$-Mediated Michael Additions from Ketone 1: Neat $\mathrm{TiCl}_{4}(235 \mu \mathrm{L}, 2.1 \mathrm{mmol})$ was carefully added to a solution of $1(192 \mathrm{mg}, 1.0 \mathrm{mmol})$ in $\mathrm{CH}_{2} \mathrm{Cl}_{2}(4 \mathrm{~mL})$ at $-78{ }^{\circ} \mathrm{C}$ under $\mathrm{N}_{2}$. The resultant yellow suspension was stirred for $5 \mathrm{~min}$ and $i$ $\mathrm{Pr}_{2} \mathrm{NEt}(195 \mu \mathrm{L}, 1.1 \mathrm{mmol})$ was added dropwise. The ensuing dark red solution was stirred for $30 \mathrm{~min}$ at $-78{ }^{\circ} \mathrm{C}$. Then, the corresponding nitroalkene $(1.2 \mathrm{mmol})$ was added and the reaction mixture was stirred at $-78{ }^{\circ} \mathrm{C}$ for $1 \mathrm{~h}$. The reaction was quenched at $-78{ }^{\circ} \mathrm{C}$ with $25 \% \mathrm{NH}_{4} \mathrm{~F}(6$ $\mathrm{mL}$ ) and stirred for $30 \mathrm{~min}$ at room temperature. The mixture was partitioned with $\mathrm{CH}_{2} \mathrm{Cl}_{2}(20 \mathrm{~mL})$ and $\mathrm{H}_{2} \mathrm{O}(20 \mathrm{~mL})$, and the aqueous layer was extracted with $\mathrm{CH}_{2} \mathrm{Cl}_{2}(3 \times 10 \mathrm{~mL})$. The combined organic layers were dried $\left(\mathrm{MgSO}_{4}\right)$ and concentrated. The residue was analyzed by ${ }^{1} \mathrm{H}$ NMR and purified by flash column chromatography to afford the corresponding Michael adduct 2.

(2S,4S,5R)-2-Benzyloxy-4-methyl-6-nitro-5-phenyl-3-hexanone (2a): Purple oil. Yield: $276 \mathrm{mg}(0.8 \mathrm{mmol}), 80 \%$; $R_{f}$ (hexanes/EtOAc 90:10) 0.20 . [a]D $+99.0\left(c\right.$ 1.0, $\left.\mathrm{CHCl}_{3}\right)$. IR (ATR): $v=3014,3082,3060,3028$, 2974, 2930, 2870, 1708, 1549, 1451, $1369 \mathrm{~cm}^{-1} .{ }^{1} \mathrm{H}$ NMR $(400 \mathrm{MHz}$, $\left.\mathrm{CDCl}_{3}\right): \delta=7.41-7.14(\mathrm{~m}, 10 \mathrm{H}), 4.64(\mathrm{~d}, J=11.7 \mathrm{~Hz}, 1 \mathrm{H}), 4.62(\mathrm{dd}, J=$ 12.6, $10.0 \mathrm{~Hz}, 1 \mathrm{H}), 4.54(\mathrm{~d}, J=11.7 \mathrm{~Hz}, 1 \mathrm{H}), 4.52(\mathrm{dd}, J=12.6,4.6 \mathrm{~Hz}$, $1 \mathrm{H}), 4.01(\mathrm{q}, J=6.7 \mathrm{~Hz}, 1 \mathrm{H}), 3.72(\mathrm{td}, J=9.9,4.6 \mathrm{~Hz}, 1 \mathrm{H}), 3.41(\mathrm{dq}, J$ 
= 9.9, 7.0 Hz, $1 \mathrm{H}), 1.32(\mathrm{~d}, J=6.7 \mathrm{~Hz}, 3 \mathrm{H}), 0.92(\mathrm{~d}, J=7.0 \mathrm{~Hz}, 3 \mathrm{H})$ ppm. ${ }^{13} \mathrm{C}$ NMR $\left(100.6 \mathrm{MHz}, \mathrm{CDCl}_{3}\right): \delta=213.5,137.6,137.3,128.9$, 128.6, 128.1, 128.0, 127.9, 127.8, 79.1, 78.2, 71.9, 46.2, 43.8, 16.3, 15.8 ppm. HRMS (ESI): calcd. for $\mathrm{C}_{20} \mathrm{H}_{23} \mathrm{NNaO}_{4}[\mathrm{M}+\mathrm{Na}]^{+} 364.1519$; found 364.1522.

(2S,4S,5S)-2-Benzyloxy-4-methyl-6-nitro-5-phenyl-3-hexanone (3a): White needles; m.p. 93-95 ${ }^{\circ} \mathrm{C}$; $R_{f}$ (hexanes/EtOAc 90:10) $0.15 .[\alpha]_{\mathrm{D}}$ +14.9 (c 1.0, $\left.\mathrm{CHCl}_{3}\right)$. IR (ATR): $v=3025,2976,2918,2874,1708,1548$, 1454, 1370, $1107 \mathrm{~cm}^{-1} .{ }^{1} \mathrm{H}$ NMR $\left(400 \mathrm{MHz}, \mathrm{CDCl}_{3}\right): \delta=7.35-7.17(\mathrm{~m}, 10$ $\mathrm{H}), 4.76(\mathrm{dd}, J=12.7,5.1 \mathrm{~Hz}, 1 \mathrm{H}), 4.69(\mathrm{dd}, J=12.7,9.8 \mathrm{~Hz}, 1 \mathrm{H}), 4.28$ $(\mathrm{d}, J=11.7 \mathrm{~Hz}, 1 \mathrm{H}), 4.10(\mathrm{~d}, J=11.7 \mathrm{~Hz}, 1 \mathrm{H}), 3.84(\mathrm{td}, J=9.8,5.1 \mathrm{~Hz}$, $1 \mathrm{H}), 3.73(\mathrm{q}, J=6.9 \mathrm{~Hz}, 1 \mathrm{H}), 3.42(\mathrm{dq}, J=9.8,6.9 \mathrm{~Hz}, 1 \mathrm{H}), 1.17(\mathrm{~d}, J=$ $6.9 \mathrm{~Hz}, 3 \mathrm{H}), 1.04(\mathrm{~d}, J=6.9 \mathrm{~Hz}, 3 \mathrm{H}) \mathrm{ppm} .{ }^{13} \mathrm{C}$ NMR $(100.6 \mathrm{MHz}$, $\left.\mathrm{CDCl}_{3}\right): \delta=212.9,138.3,137.7,129.0,128.6,128.3,128.1,128.0,127.8$ 80.6, 77.7, 71.5, 45.9, 44.5, 16.4, 14.6 ppm. HRMS (ESI): calcd. for $\mathrm{C}_{20} \mathrm{H}_{23} \mathrm{NNaO}_{4}[\mathrm{M}+\mathrm{Na}]^{+}: 364.1519 ;$ found: 364.1516 .

(2S,4S,5R)-2-Benzyloxy-4-methyl-5-(4-methylphenyl)-6-nitro-3-

hexanone (2b): Purple oil. Yield: $141 \mathrm{mg}(0.4 \mathrm{mmol}, 0.5 \mathrm{mmol}$ scale), $80 \% ; R_{f}$ (hexanes/EtOAc 90:10) 0.20. [a] $]_{\mathrm{D}}+12.1$ (c 1.0, $\left.\mathrm{CHCl}_{3}\right)$. IR (ATR): $v=3025,2976,2918,2874,1708,1548,1454,1370,1107 \mathrm{~cm}^{-1} .{ }^{1} \mathrm{H}$ NMR $\left(400 \mathrm{MHz}_{,} \mathrm{CDCl}_{3}\right): \delta=7.39-7.31(\mathrm{~m}, 5 \mathrm{H}), 7.13-7.09(\mathrm{~m}, 2 \mathrm{H})$, 7.06-7.02 (m, $2 \mathrm{H}), 4.63(\mathrm{~d}, J=11.7 \mathrm{~Hz}, 1 \mathrm{H}), 4.58(\mathrm{dd}, J=12.5,10.2 \mathrm{~Hz}$ $1 \mathrm{H}$ ), $4.54(\mathrm{~d}, J=11.7 \mathrm{~Hz}, 1 \mathrm{H}), 4.50(\mathrm{dd}, J=12.5,4.6 \mathrm{~Hz}, 1 \mathrm{H}), 4.00$ (q, $J=6.8 \mathrm{~Hz}, 1 \mathrm{H}), 3.70-3.64(\mathrm{~m}, 1 \mathrm{H}), 3.42-3.34(\mathrm{~m}, 1 \mathrm{H}), 2.31(\mathrm{~s}, 3 \mathrm{H})$, $1.32(\mathrm{~d}, J=6.8 \mathrm{~Hz}, 3 \mathrm{H}), 0.92(\mathrm{~d}, J=7.0 \mathrm{~Hz}, 3 \mathrm{H}) \mathrm{ppm} .{ }^{13} \mathrm{C}$ NMR $(100.6$ $\left.\mathrm{MHz}, \mathrm{CDCl}_{3}\right): \delta=213.7,137.7,137.5,134.6,129.7,128.7,128.2,128.0$, 128.0, 79.3, 78.5, 72.0, 46.0, 43.9, 21.2, 16.5, 15.9 ppm. HRMS (ESI): calcd. for $\mathrm{C}_{21} \mathrm{H}_{29} \mathrm{~N}_{2} \mathrm{O}_{4}\left[\mathrm{M}+\mathrm{NH}_{4}\right]^{+}: 373.2122$; found: 373.2130 .

(2S,4S,5R)-2-Benzyloxy-5-(4-methoxyphenyl)-4-methyl-6-nitro-3hexanone (2c): Brownish oil. Yield: $215 \mathrm{mg}$ (0.8 mmol), 80\%; $R_{f}$ (hexanes/EtOAc 85:15) 0.25. [ $\alpha]_{\mathrm{D}}+146.0$ (c 1.0, $\mathrm{CHCl}_{3}$ ). IR (ATR): $v=$ $3085,3057,3030,2981,2933,2870,2834,1708,1549,1450,1375 \mathrm{~cm}^{-1}$ ${ }^{1} \mathrm{H}$ NMR $\left(400 \mathrm{MHz}_{\mathrm{CDCl}}\right): \delta=7.41-7.33(\mathrm{~m}, 5 \mathrm{H}), 7.10-7.05(\mathrm{~m}, 2 \mathrm{H})$, 6.86-6.82 (m, $2 \mathrm{H}), 4.64(\mathrm{~d}, J=11.7 \mathrm{~Hz}, 1 \mathrm{H}), 4.56(\mathrm{dd}, J=12.4,4.6 \mathrm{~Hz}$, $1 \mathrm{H}), 4.54(\mathrm{~d}, J=11.7 \mathrm{~Hz}, 1 \mathrm{H}), 4.49(\mathrm{dd}, J=12.4,4.6 \mathrm{~Hz}, 1 \mathrm{H}), 4.01(\mathrm{q}$ $J=6.7 \mathrm{~Hz}, 1 \mathrm{H}), 3.78(\mathrm{~s}, 3 \mathrm{H}), 3.67(\mathrm{dt}, J=9.8,4.6 \mathrm{~Hz}, 1 \mathrm{H}), 3.37$ (dq, $J$ $=9.8,7.0 \mathrm{~Hz}, 1 \mathrm{H}), 1.32(\mathrm{~d}, J=6.7 \mathrm{~Hz}, 3 \mathrm{H}), 0.92(\mathrm{~d}, J=7.0 \mathrm{~Hz}, 3 \mathrm{H})$ ppm. ${ }^{13} \mathrm{C}$ NMR $\left(100.6 \mathrm{MHz}, \mathrm{CDCl}_{3}\right): \delta=213.7,159.1,137.3,129.4$, $129.1,128.6,128.1,127.9,79.2,78.4,71.8,55.2,45.5,43.9,16.3,15.8$ ppm. HRMS (ESI): calcd. for $\mathrm{C}_{21} \mathrm{H}_{25} \mathrm{NNaO}_{5}[\mathrm{M}+\mathrm{Na}]^{+:} 394.1625$, found: 394.1636 .

(2S,4S,5R)-2-Benzyloxy-4-methyl-5-(3,4-methylenedioxyphenyl)-6nitro-3-hexanone (2d): Yellowish oil. Yield: $157 \mathrm{mg}(0.41 \mathrm{mmol}, 0.5$

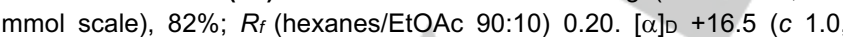
$\mathrm{CHCl}_{3}$ ). IR (ATR): $v=3025,2985,2927,2900,2869,1712,1543,1499$, 1485, 1441, 1370, 1241, $1031 \mathrm{~cm}^{-1} .{ }^{1} \mathrm{H}$ NMR $\left(400 \mathrm{MHz}, \mathrm{CDCl}_{3}\right): \delta=$ 7.41-7.31 (m, $5 \mathrm{H}), 6.75-6.73(\mathrm{~m}, 1 \mathrm{H}), 6.63-6.60(\mathrm{~m}, 2 \mathrm{H}), 5.95(\mathrm{~s}, 2 \mathrm{H})$, $4.66(\mathrm{~d}, J=11.7 \mathrm{~Hz}, 1 \mathrm{H}), 4.54(\mathrm{~d}, J=11.7 \mathrm{~Hz}, 1 \mathrm{H}), 4.51(\mathrm{dd}, J=12.5$, $9.9 \mathrm{~Hz}, 1 \mathrm{H}), 4.46(\mathrm{dd}, J=12.5,4.9 \mathrm{~Hz}, 1 \mathrm{H}), 4.02(\mathrm{q}, J=6.7 \mathrm{~Hz}, 1 \mathrm{H})$, $3.63(\mathrm{td}, J=9.9,4.9 \mathrm{~Hz}, 1 \mathrm{H}), 3.33(\mathrm{dq}, J=9.9,7.0 \mathrm{~Hz}, 1 \mathrm{H}), 1.33(\mathrm{~d}, J=$ $6.7 \mathrm{~Hz}, 3 \mathrm{H}$ ), $0.92(\mathrm{~d}, J=7.0 \mathrm{~Hz}, 3 \mathrm{H}) \mathrm{ppm} .{ }^{13} \mathrm{C} \mathrm{NMR}(100.6 \mathrm{MHz}$, $\left.\mathrm{CDCl}_{3}\right): \delta=213.6,148.2,147.3,137.4,131.3,128.8,128.3,128.1,121.8$ 108.7, 108.1, 101.3, 79.2, 78.6, 72.0, 46.1, 44.0, 16.4, 15.9 ppm. HRMS (ESI): calcd. for $\mathrm{C}_{21} \mathrm{H}_{27} \mathrm{~N}_{2} \mathrm{O}_{6}\left[\mathrm{M}+\mathrm{NH}_{4}\right]^{+}$: 403.1864 ; found: 403.1873 .

(2S,4S,5R)-2-Benzyloxy-5-(4-chlorophenyl)-4-methyl-6-nitro-3hexanone (2e): Yellowish oil. Yield: $262 \mathrm{mg}(0.7 \mathrm{mmol}), 70 \% ; R_{f}$ (hexanes/EtOAc 85:15) 0.30. [ $\alpha]_{\mathrm{D}}+42.0$ (c 1.0, $\mathrm{CHCl}_{3}$ ). IR (ATR): $v 3083$, $3063,3030,2977,2928,2874,1708,1549,1490,1370 \mathrm{~cm}^{-1}$. ${ }^{1} \mathrm{H}$ NMR $\left(400 \mathrm{MHz}, \mathrm{CDCl}_{3}\right): \delta=7.44-7.27(\mathrm{~m}, 7 \mathrm{H}), 7.12-7.08(\mathrm{~m}, 2 \mathrm{H}), 4.67(\mathrm{~d}, J$
$=11.6 \mathrm{~Hz}, 1 \mathrm{H}), 4.56(\mathrm{dd}, J=12.7,10.0 \mathrm{~Hz}, 1 \mathrm{H}), 4.54(\mathrm{~d}, J=11.6 \mathrm{~Hz}, 1$ $\mathrm{H}), 4.50(\mathrm{dd}, J=12.7,4.7 \mathrm{~Hz}, 1 \mathrm{H}), 4.02(\mathrm{q}, J=6.7 \mathrm{~Hz}, 1 \mathrm{H}), 3.70(\mathrm{td}, J=$ 10.0, $4.7 \mathrm{~Hz}, 1 \mathrm{H}), 3.38(\mathrm{dq}, J=10.0,7.0 \mathrm{~Hz}, 1 \mathrm{H}), 1.32(\mathrm{~d}, J=6.7 \mathrm{~Hz}, 3$ $\mathrm{H}), 0.90(\mathrm{~d}, J=7.0 \mathrm{~Hz}, 3 \mathrm{H}) \mathrm{ppm} .{ }^{13} \mathrm{C} \mathrm{NMR}\left(100.6 \mathrm{MHz}, \mathrm{CDCl}_{3}\right): \delta 213.2$, 137.2, 136.1, 133.7, 129.4, 128.1, 128.6, 128.2, 128.0, 78.9, 78.1, 71.9, 45.6, 43.6, 16.2, 15.7 ppm. HRMS (ESI): calcd. for $\mathrm{C}_{20} \mathrm{H}_{22} \mathrm{CINNaO}_{4}[\mathrm{M}+$ $\mathrm{Na}]^{+}:$398.113; found: 398.1133

\section{(2S,4S,5S)-2-Benzyloxy-5-furyl-4-methyl-6-nitro-3-hexanone}

$(2 g)$ : Brown needles. Yield: $104 \mathrm{mg}(0.32 \mathrm{mmol}, 0.5 \mathrm{mmol}$ scale), $64 \%$; m.p.

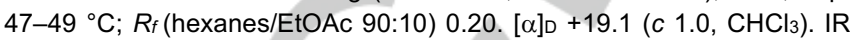
(ATR): $v=2980,2936,2860,1717,1548,1508,1450,1365,1094 \mathrm{~cm}^{-1}$. ${ }^{1} \mathrm{H}$ NMR $\left(400 \mathrm{MHz}, \mathrm{CDCl}_{3}\right): \delta 7.39-7.30(\mathrm{~m}, 6 \mathrm{H}), 6.28(\mathrm{dd}, J=3.2,1.9$ $\mathrm{Hz}, 1 \mathrm{H}), 6.18-6.16(\mathrm{~m}, 1 \mathrm{H}), 4.66(\mathrm{dd}, J=12.7,9.9 \mathrm{~Hz}, 1 \mathrm{H}), 4.62(\mathrm{~d}, J=$ $11.7 \mathrm{~Hz}, 1 \mathrm{H}), 4.54(\mathrm{~d}, J=11.8 \mathrm{~Hz}, 1 \mathrm{H}), 4.49(\mathrm{dd}, J=12.7,4.4 \mathrm{~Hz}, 1 \mathrm{H})$, $4.01(\mathrm{q}, J=6.8 \mathrm{~Hz}, 1 \mathrm{H}), 3.95-3.89(\mathrm{~m}, 1 \mathrm{H}), 3.54-3.47(\mathrm{~m}, 1 \mathrm{H}), 1.33(\mathrm{~d}$, $J=6.8 \mathrm{~Hz}, 3 \mathrm{H}), 1.01(\mathrm{~d}, \mathrm{~J}=7.1 \mathrm{~Hz}, 3 \mathrm{H}) \mathrm{ppm} .{ }^{13} \mathrm{C}$ NMR $(100.6 \mathrm{MHz}$, $\left.\mathrm{CDCl}_{3}\right): \delta=213.0,150.9,142.6,137.5,128.7,128.2,128.0,110.5,108.9$ $(\mathrm{CH}), 79.3,76.3,71.9,42.4,40.0,16.1,15.6$ ppm. HRMS (ESI): calcd. for $\mathrm{C}_{18} \mathrm{H}_{25} \mathrm{~N}_{2} \mathrm{O}_{5}\left[\mathrm{M}+\mathrm{NH}_{4}\right]^{+}: 349.1758$; found: 349.1760

General Experimental Procedure for the $\mathrm{SnCl}_{4}$-Mediated Michael Additions from Ketone 1: Neat $\mathrm{TiCl}_{4}(61 \mu \mathrm{L}, 0.55 \mathrm{mmol})$ was carefully added to a solution of 1 (96 $\mathrm{mg}, 0.5 \mathrm{mmol})$ in $\mathrm{CH}_{2} \mathrm{Cl}_{2}(2 \mathrm{~mL})$ at $-78{ }^{\circ} \mathrm{C}$. The resultant yellow suspension was stirred for 5 min and $i-\operatorname{Pr}_{2} \mathrm{NEt}(96 \mu \mathrm{L}$, $0.55 \mathrm{mmol}$ ) was added dropwise. The ensuing dark red solution was stirred for $30 \mathrm{~min}$ at $-78{ }^{\circ} \mathrm{C}$. Then, $1 \mathrm{M} \mathrm{SnCl}_{4}$ in $\mathrm{CH}_{2} \mathrm{Cl}_{2}(0.55 \mathrm{~mL} \mu \mathrm{L}, 0.55$ $\mathrm{mmol}$ ) was added, followed $5 \mathrm{~min}$ later by the corresponding nitroalkene $(0.6 \mathrm{mmol})$ and the resultant mixture was stirred at $-78{ }^{\circ} \mathrm{C}$ for $3 \mathrm{~h}$. The reaction was quenched at $-78{ }^{\circ} \mathrm{C}$ with $25 \% \mathrm{NH}_{4} \mathrm{~F}(3 \mathrm{~mL})$ and stirred for $30 \mathrm{~min}$ at room temperature with vigorous stirring The mixture was partitioned with $\mathrm{CH}_{2} \mathrm{Cl}_{2}(10 \mathrm{~mL})$ and $\mathrm{H}_{2} \mathrm{O}(10 \mathrm{~mL})$, the aqueous layer was extracted with $\mathrm{CH}_{2} \mathrm{Cl}_{2}(3 \times 10 \mathrm{~mL})$. The combined organic layers were dried $\left(\mathrm{MgSO}_{4}\right)$ and concentrated. The residue was analyzed by ${ }^{1} \mathrm{H}$ NMR and purified by flash column chromatography to afford the corresponding Michael adduct 2

(2S,4S,5S)-2-Benzyloxy-4-methyl-5-nitromethyl-7-phenyl-3-

heptanone (2h): Yellowish oil. Yield: $99 \mathrm{mg}(0.27 \mathrm{mmol}), 54 \% ; R_{f}$ (hexanes/EtOAc 90:10) 0.10. [ $\alpha]_{\mathrm{D}}+164.0$ (c 1.0, $\left.\mathrm{CHCl}_{3}\right)$. IR (ATR): $\mathrm{v}=$ $3083,3057,3025,2976,2927,2860,1708,1544,1450,1375,1112 \mathrm{~cm}^{-1}$. ${ }^{1} \mathrm{H}$ NMR $\left(400 \mathrm{MHz}, \mathrm{CDCl}_{3}\right): \delta=7.39-7.09(\mathrm{~m}, 10 \mathrm{H}), 4.57(\mathrm{~d}, \mathrm{~J}=11.7 \mathrm{~Hz}$, $1 \mathrm{H}), 4.53(\mathrm{~d}, J=11.7 \mathrm{~Hz}, 1 \mathrm{H}), 4.52(\mathrm{dd}, J=12.7,4.4 \mathrm{~Hz}, 1 \mathrm{H}), 4.29(\mathrm{dd}$, $J=12.7,8.0 \mathrm{~Hz}, 1 \mathrm{H}$ ), $3.99(\mathrm{q}, J=6.8 \mathrm{~Hz}, 1 \mathrm{H}), 3.28(\mathrm{qd}, J=7.1,5.1 \mathrm{~Hz}$ $1 \mathrm{H}), 2.65-2.55(\mathrm{~m}, 2 \mathrm{H}), 2.63(\mathrm{t}, J=8.1 \mathrm{~Hz}, 2 \mathrm{H}), 1.36(\mathrm{~d}, J=6.8 \mathrm{~Hz}, 3$ $\mathrm{H}), 1.03(\mathrm{~d}, J=7.1 \mathrm{~Hz}, 3 \mathrm{H}) \mathrm{ppm} .{ }^{13} \mathrm{C}$ NMR (100.6 MHz, $\left.\mathrm{CDCl}_{3}\right): \delta$ $=213.6,140.7,137.3,128.5,128.5$ 128.2, 128.0, 127.8, 126.2, 79.4, $76.4,71.7,41.2,38.1,33.1,32.0,16.7,11.7$ ppm. HRMS (ESI): calcd. for $\mathrm{C}_{22} \mathrm{H}_{27} \mathrm{NNaO}_{4}[\mathrm{M}+\mathrm{Na}]^{+}:$392.1832; found: 392.1839 .

(2S,4S,5S)-2-Benzyloxy-4-methyl-5-nitromethyl-3-octanone (2i): Yellowish oil. Yield: $105 \mathrm{mg}(0.34 \mathrm{mmol}), 68 \% ; R_{f}$ (hexanes/EtOAc 85:15) 0.10. $[\alpha]_{\mathrm{D}}+22.9\left(c 1.0, \mathrm{CHCl}_{3}\right)$. IR (ATR): $v=2963,2932,2869,1708$,

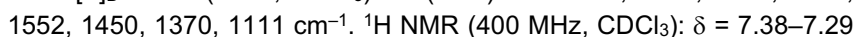
$(\mathrm{m}, 5 \mathrm{H}), 4.60(\mathrm{~d}, J=11.8 \mathrm{~Hz}, 1 \mathrm{H}), 4.56(\mathrm{~d}, J=11.8 \mathrm{~Hz}, 1 \mathrm{H}), 4.49(\mathrm{dd}, J$ $=12.6,4.3 \mathrm{~Hz}, 1 \mathrm{H}), 4.24(\mathrm{dd}, J=12.6,8.2 \mathrm{~Hz}, 1 \mathrm{H}), 4.04(\mathrm{q}, J=6.8 \mathrm{~Hz}$, $1 \mathrm{H}), 3.28-3.23(\mathrm{~m}, 1 \mathrm{H}), 2.61-2.54(\mathrm{~m}, 1 \mathrm{H}), 1.39(\mathrm{~d}, J=6.8 \mathrm{~Hz}, 3 \mathrm{H})$, 1.38-1.31 (m, $4 \mathrm{H}), 1.01(\mathrm{~d}, J=7.1 \mathrm{~Hz}, 3 \mathrm{H}), 0.89-0.85(\mathrm{~m}, 3 \mathrm{H}) \mathrm{ppm}$. ${ }^{13} \mathrm{C}$ NMR $\left(100.6 \mathrm{MHz}, \mathrm{CDCl}_{3}\right): \delta=214.1,137.5,128.7,128.1,128.0$, $79.7,76.8,71.9,41.3,38.3,32.7,20.1,17.0,13.9,11.7$ ppm. HRMS (ESI): calcd. for $\mathrm{C}_{17} \mathrm{H}_{29} \mathrm{~N}_{2} \mathrm{O}_{4}\left[\mathrm{M}+\mathrm{NH}_{4}\right]^{+}$: 325.2122; found: 325.2118 .

(2S,4S,5S)-2-Benzyloxy-4,7-dimethyl-5-nitromethyl-3-octanone (2j): Yellowish oil. Yield: $128 \mathrm{mg}(0.40 \mathrm{mmol}), 80 \%$; $R_{f}$ (hexanes/EtOAc 90:10) 
0.25. $[\alpha]_{\mathrm{D}}+21.4\left(\mathrm{c} 1.0, \mathrm{CHCl}_{3}\right)$. IR (ATR): $v=3029,2954,2932,2865$, $1708,1552,1450,1378,1111 \mathrm{~cm}^{-1} .{ }^{1} \mathrm{H}$ NMR $\left(400 \mathrm{MHz}, \mathrm{CDCl}_{3}\right): \delta=$ 7.38-7.28 (m, $5 \mathrm{H}), 4.59(\mathrm{~d}, J=11.8 \mathrm{~Hz}, 1 \mathrm{H}), 4.56(\mathrm{~d}, J=11.8 \mathrm{~Hz}, 1 \mathrm{H})$, 4.47 (dd, $J=12.6,4.3 \mathrm{~Hz}, 1 \mathrm{H}$ ), 4.21 (dd, $J=12.6,8.1 \mathrm{~Hz}, 1 \mathrm{H}$ ), 4.03 (q, $\mathrm{J}=6.8 \mathrm{~Hz}, 1 \mathrm{H}), 3.26-3.19(\mathrm{~m}, 1 \mathrm{H}), 2.67-2.60(\mathrm{~m}, 1 \mathrm{H}), 1.65-1.55(\mathrm{~m}, 1$ $\mathrm{H}), 1.40(\mathrm{~d}, J=6.8 \mathrm{~Hz}, 3 \mathrm{H}), 1.30-1.17(\mathrm{~m}, 2 \mathrm{H}), 1.00(\mathrm{~d}, J=7.0 \mathrm{~Hz}, 3 \mathrm{H})$ $0.88(\mathrm{~d}, J=6.6 \mathrm{~Hz}, 3 \mathrm{H}), 0.82(\mathrm{~d}, J=6.6 \mathrm{~Hz}, 3 \mathrm{H}) \mathrm{ppm} .{ }^{13} \mathrm{C}$ NMR $(100.6$ $\left.\mathrm{MHz}, \mathrm{CDCl}_{3}\right): \delta=214.2,137.5,128.7,128.2,128.1,79.7,77.0,71.9$, 41.4, 39.7, 36.2, 25.2, 22.8, 22.2, 17.1, 11.4 ppm. HRMS (ESI): calcd. for $\mathrm{C}_{18} \mathrm{H}_{31} \mathrm{~N}_{2} \mathrm{O}_{4}\left[\mathrm{M}+\mathrm{NH}_{4}\right]^{+}:$339.2278; found: 339.2276

\section{(2S,4S,5S)-2-Benzyloxy-4,6-dimethyl-5-nitromethyl-3-heptanone}

(2k): Yellowish oil. Yield: $44 \mathrm{mg}(0.14 \mathrm{mmol}), 28 \% ; R_{f}$ (hexanes/EtOAc 90:10) 0.30. $[\alpha]_{\mathrm{D}}+15.9\left(c\right.$ 1.0, $\left.\mathrm{CHCl}_{3}\right)$. IR (ATR): $v=3025,2963,2932$, 2883, 1708, 1543, 1454, 1374, $1107 \mathrm{~cm}^{-1}$. ${ }^{1} \mathrm{H}$ NMR $\left(400 \mathrm{MHz}, \mathrm{CDCl}_{3}\right): \delta$ $=7.39-7.29(\mathrm{~m}, 5 \mathrm{H}), 4.61(\mathrm{~d}, J=11.8 \mathrm{~Hz}, 1 \mathrm{H}), 4.56(\mathrm{~d}, J=11.8 \mathrm{~Hz}, 1$ H), $4.45(\mathrm{dd}, J=13.8,4.4 \mathrm{~Hz}, 1 \mathrm{H}), 4.29(\mathrm{dd}, J=13.8,7.1 \mathrm{~Hz}, 1 \mathrm{H}), 4.06$ (q, $J=6.8 \mathrm{~Hz}, 1 \mathrm{H}), 3.39-3.33(\mathrm{~m}, 1 \mathrm{H}), 2.61-2.55(\mathrm{~m}, 1 \mathrm{H}), 1.74-1.66$ $(\mathrm{m}, 1 \mathrm{H}), 1.41(\mathrm{~d}, J=6.8 \mathrm{~Hz}, 3 \mathrm{H}), 0.97(\mathrm{~d}, J=7.0 \mathrm{~Hz}, 3 \mathrm{H}), 0.96(\mathrm{~d}, J=$ $6.8 \mathrm{~Hz}, 3 \mathrm{H}$ ), $0.87(\mathrm{~d}, J=6.8 \mathrm{~Hz}, 3 \mathrm{H}) \mathrm{ppm} .{ }^{13} \mathrm{C}$ NMR $(100.6 \mathrm{MHz}$, $\left.\mathrm{CDCl}_{3}\right): \delta=214.2,137.6,128.7,128.1,128.0,79.5,75.1,71.8,43.5$, 40.5, 30.1, 20.6, 19.2, 17.1, $12.1 \mathrm{ppm}$. HRMS (ESI): calcd. for $\mathrm{C}_{17} \mathrm{H}_{29} \mathrm{~N}_{2} \mathrm{O}_{4}\left[\mathrm{M}+\mathrm{NH}_{4}\right]^{+}: 325.2122$; found: 325.2125 .

(2S,4S,5S)-2,7-Dibenzyloxy-4-methyl-5-nitromethyl-3-hepanone (2I): Colorless oil. Yield: $51 \mathrm{mg}(0.13 \mathrm{mmol}), 25 \% ; R_{f}$ (hexanes/EtOAc 80:20) 0.20. $[\alpha]_{\mathrm{D}}+10.2\left(c \mathrm{1} .0, \mathrm{CHCl}_{3}\right)$. IR (ATR): $v=3025,2972,2927,2856$ 1717, 1548, 1459, 1378, $1094 \mathrm{~cm}^{-1} .{ }^{1} \mathrm{H}$ NMR $\left(400 \mathrm{MHz}, \mathrm{CDCl}_{3}\right): \delta=$ 7.38-7.26 (m, $5 \mathrm{H}), 4.55(\mathrm{~s}, 2 \mathrm{H}), 4.52(\mathrm{dd}, J=13.0,4.6 \mathrm{~Hz}, 1 \mathrm{H}), 4.42(\mathrm{~d}$ $J=11.8 \mathrm{~Hz}, 1 \mathrm{H}), 4.38(\mathrm{~d}, J=11.8 \mathrm{~Hz}, 1 \mathrm{H}), 4.36(\mathrm{dd}, J=13.0,7.7 \mathrm{~Hz}, 1$ $\mathrm{H}), 4.04(\mathrm{q}, J=6.8 \mathrm{~Hz}, 1 \mathrm{H}), 3.49(\mathrm{t}, J=6.0 \mathrm{~Hz}, 2 \mathrm{H}), 3.30-3.24(\mathrm{~m}, 1 \mathrm{H})$, 2.77-2.69 (m, $1 \mathrm{H}$ ), 1.71 (ddd, $J=12.5,6.1,2.4 \mathrm{~Hz}, 2 \mathrm{H}$ ), 1.34 (d, $J=6.8$ $\mathrm{Hz}, 3 \mathrm{H}), 1.03$ (d, J = 7.0 Hz, $3 \mathrm{H}$ ) ppm. ${ }^{13} \mathrm{C} \mathrm{NMR}\left(100.6 \mathrm{MHz}, \mathrm{CDCl}_{3}\right): \delta$ $=213.8,138.1,137.6,128.6,128.5,128.1,128.0,127.8,79.4,76.6,73.1$ 71.8, 67.9, 41.8, 36.6, 30.3, 16.8, 12.2 ppm. HRMS (ESI): calcd. for $\mathrm{C}_{23} \mathrm{H}_{33} \mathrm{~N}_{2} \mathrm{O}_{5}\left[\mathrm{M}+\mathrm{NH}_{4}\right]^{+}:$417.2384; found: 417.2393 .

\section{(2S,4S,5S)-2-Benzyloxy-4-methyl-5-nitromethyl-7-}

triisopropylsilyloxy-3-heptanone $(\mathbf{2 m})$ : Colorless oil. Yield: $133 \mathrm{mg}$

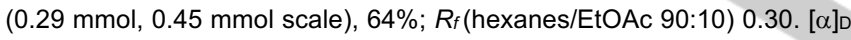
$+11.6\left(c\right.$ 1.0, $\left.\mathrm{CHCl}_{3}\right)$. IR (ATR): $v=2942,2860,1710,1546,1457,1372$, $1096 \mathrm{~cm}^{-1} .{ }^{1} \mathrm{H} \mathrm{NMR}\left(400 \mathrm{MHz}, \mathrm{CDCl}_{3}\right): \delta=7.40-7.20(\mathrm{~m}, 5 \mathrm{H}), 4.56(\mathrm{dd}$, $J=12.9,5.0 \mathrm{~Hz}, 1 \mathrm{H}), 4.56(\mathrm{~s}, 2 \mathrm{H}), 4.40(\mathrm{dd}, J=12.9,7.3 \mathrm{~Hz}, 1 \mathrm{H}), 4.07$ (q, $J=6.8 \mathrm{~Hz}, 1 \mathrm{H}), 3.75(\mathrm{t}, J=5.9 \mathrm{~Hz}, 2 \mathrm{H}), 3.30(\mathrm{dq}, J=7.0,5.2 \mathrm{~Hz}, 1$ H), $2.79-2.70(\mathrm{~m}, 1 \mathrm{H}), 1.67-1.50(\mathrm{~m}, 2 \mathrm{H}), 1.37(\mathrm{~d}, J=6.8 \mathrm{~Hz}, 3 \mathrm{H})$, $1.05(\mathrm{~d}, J=7.0 \mathrm{~Hz}, 3 \mathrm{H}), 1.10-1.00(\mathrm{~m}, 21 \mathrm{H}) \mathrm{ppm} .{ }^{13} \mathrm{C} \mathrm{NMR}(100.6 \mathrm{MHz}$ $\left.\mathrm{CDCl}_{3}\right): \delta=213.6,137.5,128.5,127.9,79.3,76.6,71.6,61.1,41.5,36.3$, 32.9, 17.9, 16.7, 12.3, 11.8 ppm. HRMS (ESI): calcd. for $\mathrm{C}_{25} \mathrm{H}_{43} \mathrm{NNaO}_{5} \mathrm{Si}$ $[\mathrm{M}+\mathrm{Na}]^{+}:$488.2803; found: 488.2806 .

\section{(2S,4S,5R)-2-Benzyloxy-4-methyl-6-nitro-1,5-diphenyl-3-hexanone}

(6a): Neat $\mathrm{TiCl}_{4}(61 \mu \mathrm{L}, 0.55 \mathrm{mmol})$ was carefully added to a solution of chiral ketone 4 (134 mg, $0.5 \mathrm{mmol})$ in $\mathrm{CH}_{2} \mathrm{Cl}_{2}(2 \mathrm{~mL})$ at $-78^{\circ} \mathrm{C}$ under $\mathrm{N}_{2}$ and the resultant yellow suspension was stirred for $5 \mathrm{~min}$. $i-\operatorname{Pr}_{2} \mathrm{NEt}(96 \mu \mathrm{L}$, $0.55 \mathrm{mmol}$ ) was added dropwise and the ensuing dark solution was stirred for $30 \mathrm{~min}$ at $-78{ }^{\circ} \mathrm{C}$. Then, $\mathrm{TiCl}_{4}(61 \mu \mathrm{L}, 0.55 \mathrm{mmol})$ was added dropwise, followed $10 \mathrm{~min}$ later by the addition of (E)- $\beta$-nitrostyrene (a) $(89 \mathrm{mg}, 0.6 \mathrm{mmol})$. The resultant mixture was stirred at $-78^{\circ} \mathrm{C}$ for $1 \mathrm{~h}$. The reaction was quenched at $-78{ }^{\circ} \mathrm{C}$ with a $25 \% \mathrm{NH}_{4} \mathrm{~F}(2 \mathrm{~mL})$ and stirred for $30 \mathrm{~min}$ at room temperature with vigorous stirring. The mixture was diluted with $\mathrm{CH}_{2} \mathrm{Cl}_{2}(10 \mathrm{~mL})$ and $\mathrm{H}_{2} \mathrm{O}(10 \mathrm{~mL})$. The aqueous layer was extracted with $\mathrm{CH}_{2} \mathrm{Cl}_{2}(3 \times 20 \mathrm{~mL})$ and the organic extracts were dried over $\mathrm{MgSO}_{4}$, filtered, and concentrated. The resulting crude product was purified by column chromatography (hexanes/EtOAc 90:10) to afford
$151 \mathrm{mg}(0.36 \mathrm{mmol}, 72 \%$ yield) of (2S,4S,5R)-2-benzyloxy-4-methyl-6nitro-1,5-diphenyl-3-hexanone $(\mathbf{6 a})$ as a purple oil. $R_{f}$ (hexanes/ EtOAc 90:10) 0.20. [ $\alpha]_{\mathrm{D}}-8.4\left(c \mathrm{1} .0, \mathrm{CHCl}_{3}\right.$ ). IR (ATR): $v=3081,3062,3024$, 2926, 2872, 1707, 1549,1492, 1454, 1372, 1087, 732, $697 \mathrm{~cm}^{-1} .{ }^{1} \mathrm{H}$ NMR $\left(400 \mathrm{MHz}, \mathrm{CDCl}_{3}\right): \delta=7.35-7.05(\mathrm{~m}, 15 \mathrm{H}), 4.56(\mathrm{~d}, J=11.4 \mathrm{~Hz}, 1$ H), $4.46(\mathrm{~d}, J=11.4 \mathrm{~Hz}, 1 \mathrm{H}), 4.17(\mathrm{dd}, J=6.8,4.5 \mathrm{~Hz}, 1 \mathrm{H}), 4.14(\mathrm{~d}, J=$ $10.7 \mathrm{~Hz}, 1 \mathrm{H}$ ), 4.07 (dd, $J=10.7,4.5 \mathrm{~Hz}, 1 \mathrm{H}), 3.64(\mathrm{td}, J=10.3,4.5 \mathrm{~Hz}$, $1 \mathrm{H}), 3.22(\mathrm{dq}, J=10.3,7.0 \mathrm{~Hz}, 1 \mathrm{H}), 3.15$ (dd, $J=14.1,4.5 \mathrm{~Hz}, 1 \mathrm{H}$ ), $2.94(\mathrm{dd}, J=14.1,6.8 \mathrm{~Hz}, 1 \mathrm{H}), 0.78(\mathrm{~d}, J=7.0 \mathrm{~Hz}, 3 \mathrm{H}) \mathrm{ppm} .{ }^{13} \mathrm{C}$ NMR $\left(100.6 \mathrm{MHz}, \mathrm{CDCl}_{3}\right): \delta=212.9,137.5,136.9,136.8,129.9,128.8,128.6$, $128.5,128.2,128.2,128.0,127.8,126.9,83.9,77.8,73.1,45.8,44.3$, 36.4, 15.9 ppm. HRMS (ESI): calcd. for $\mathrm{C}_{26} \mathrm{H}_{31} \mathrm{~N}_{2} \mathrm{O}_{4}\left[\mathrm{M}+\mathrm{NH}_{4}\right]^{+}$: 435.2278; found: 435.2279 .

\section{(2R,3S,5S)-5-Benzyloxy-3,6-dimethyl-1-nitro-2-phenyl-4-heptanone}

(7a): Neat $\mathrm{TiCl}_{4}(97 \mu \mathrm{L}, 0.88 \mathrm{mmol})$ was carefully added to a solution of chiral ketone $5(176 \mathrm{mg}, 0.8 \mathrm{mmol})$ in $\mathrm{CH}_{2} \mathrm{Cl}_{2}(3.2 \mathrm{~mL})$ at $-78{ }^{\circ} \mathrm{C}$ under $\mathrm{N}_{2}$ and the resultant yellow suspension was stirred for 5 min. $i-\mathrm{Pr}_{2} \mathrm{NEt}$ $(153 \mu \mathrm{L}, 0.88 \mathrm{mmol}$ ) was added dropwise and the ensuing dark solution was stirred for $30 \mathrm{~min}$ at $-78{ }^{\circ} \mathrm{C}$. Then, $\mathrm{TiCl}_{4}(97 \mu \mathrm{L}, 0.88 \mathrm{mmol})$ was added dropwise, followed $10 \mathrm{~min}$ later by the addition of $(E)-\beta$ nitrostyrene (a) (143 $\mathrm{mg}, 0.96 \mathrm{mmol})$. The resultant mixture was stirred at $-78^{\circ} \mathrm{C}$ for $1 \mathrm{~h}$. The reaction was quenched at $-78{ }^{\circ} \mathrm{C}$ with a $25 \% \mathrm{NH}_{4} \mathrm{~F}(4$ $\mathrm{mL}$ ) and stirred for $30 \mathrm{~min}$ at room temperature with vigorous stirring. The mixture was diluted with $\mathrm{CH}_{2} \mathrm{Cl}_{2}(20 \mathrm{~mL})$ and $\mathrm{H}_{2} \mathrm{O}(20 \mathrm{~mL})$. The aqueous layer was extracted with $\mathrm{CH}_{2} \mathrm{Cl}_{2}(3 \times 20 \mathrm{~mL})$ and the organic extracts were dried over $\mathrm{MgSO}_{4}$, filtered, and concentrated. The resulting crude product was purified by column chromatography (from hexanes/EtOAc $95: 5$ to $90: 10)$ to afford $173 \mathrm{mg}(0.47 \mathrm{mmol}, 59 \%$ yield $)$ of $(2 R, 3 S, 5 S)-5-$ benzyloxy-3,6-dimethyl-1-nitro-2-phenyl-4-heptanone (7a) as a brownish oil. $R_{f}$ (hexanes/ EtOAc 90:10) 0.25. [ $\left.\alpha\right]_{\mathrm{D}}+11.5$ (c 1.0, $\mathrm{CHCl}_{3}$ ). IR (ATR): $v=3024,2961,2936,2869,1707,1564,1451,1378,1064,729,697$ $\mathrm{cm}^{-1}$. ${ }^{1} \mathrm{H}$ NMR $\left(400 \mathrm{MHz}, \mathrm{CDCl}_{3}\right): \delta=7.40-7.22(\mathrm{~m}, 8 \mathrm{H}), 7.17-7.10(\mathrm{~m}$, $2 \mathrm{H}), 4.60(\mathrm{~d}, J=11.5 \mathrm{~Hz}, 1 \mathrm{H}), 4.56(\mathrm{dd}, J=12.6,10.3 \mathrm{~Hz}, 1 \mathrm{H}), 4.49(\mathrm{~d}, J$ $=11.5 \mathrm{~Hz}, 1 \mathrm{H}), 4.48(\mathrm{dd}, J=12.6,4.5 \mathrm{~Hz}, 1 \mathrm{H}), 3.70(\mathrm{td}, J=9.4,5.4 \mathrm{~Hz}$, $1 \mathrm{H}), 3.66(\mathrm{~d}, J=4.8 \mathrm{~Hz}, 1 \mathrm{H}), 3.19(\mathrm{dq}, J=9.4,6.9 \mathrm{~Hz}, 1 \mathrm{H}), 2.22-2.10(\mathrm{~m}$, $1 \mathrm{H}), 0.98(\mathrm{~d}, J=6.8 \mathrm{~Hz}, 3 \mathrm{H}), 0.95(\mathrm{~d}, J=6.9 \mathrm{~Hz}, 3 \mathrm{H}), 0.93(\mathrm{~d}, J=6.9 \mathrm{~Hz}$, $3 \mathrm{H}) \mathrm{ppm} .{ }^{13} \mathrm{C}$ NMR $\left(100.6 \mathrm{MHz}, \mathrm{CDCl}_{3}\right): \delta=213.0,137.5,137.4,128.9$, 128.5, 128.1, 128.0, 127.9, 127.9, 89.0, 77.7, 73.9, 46.6, 44.8, 30.0, 19.6, 17.2, 16.4 ppm. HRMS (ESI): calcd. for $\mathrm{C}_{22} \mathrm{H}_{31} \mathrm{~N}_{2} \mathrm{O}_{4}\left[\mathrm{M}+\mathrm{NH}_{4}\right]^{+}$: 387.2278; found: 387.2281 .

(3R,4S)-5-[(S)-1-Benzyloxyethyl]-4-methyl-3-phenyl-3,4-dihydro-2Hpyrrole (8): Solid $\mathrm{NaBH}_{4}(6 \mathrm{mg}, 0.15 \mathrm{mmol})$ was added in one portion to a mixture of $\mathrm{NiCl}_{2} \cdot 6 \mathrm{H}_{2} \mathrm{O}(12 \mathrm{mg}, 50 \mu \mathrm{mol})$ in $\mathrm{MeOH}(2 \mathrm{~mL})$ at room temperature, which produced the formation of black clumps and heavy frothing. The mixture was sonicated for $30 \mathrm{~min}$ and the resultant black dispersion was cooled to $0{ }^{\circ} \mathrm{C}$. The subsequent addition of a solution of Michael adduct 2a (34 mg, $0.1 \mathrm{mmol})$ in $\mathrm{MeOH}(2 \mathrm{~mL})$ followed by solid $\mathrm{NaBH}_{4}(19 \mathrm{mg}, 0.5 \mathrm{mmol})$ in one portion caused more frothing. The reaction mixture was stirred at $0{ }^{\circ} \mathrm{C}$ for $30 \mathrm{~min}$. Then, this was quenched by the addition of a saturated solution of $\mathrm{NH}_{4} \mathrm{Cl}(5 \mathrm{~mL})$ with vigorous stirring. It was partitioned in $\mathrm{CH}_{2} \mathrm{Cl}_{2}$ and $\mathrm{H}_{2} \mathrm{O}$. The aqueous layer was extracted with $\mathrm{CH}_{2} \mathrm{Cl}_{2}(3 \times 10 \mathrm{~mL})$ and the combined organic extracts were dried $\left(\mathrm{MgSO}_{4}\right)$, filtered, and concentrated. Eventually, the residue was purified by flash column chromatography $\left(\mathrm{CH}_{2} \mathrm{Cl}_{2} / \mathrm{MeOH}\right.$ 96:4) to afford $26 \mathrm{mg}(90 \mu \mathrm{mol}, 90 \%$ yield) of $(3 R, 4 S)-5-[(S)$-1-benzyloxyethyl]-4methyl-3-phenyl-3,4-dihydro-2H-pyrrole (8) as a colorless oil. $R_{f}$ $\left(\mathrm{CH}_{2} \mathrm{Cl}_{2} / \mathrm{MeOH}\right.$ 96:4) 0.5. $[\alpha]_{\mathrm{D}}+4.3$ (c 1.1, $\mathrm{CHCl}_{3}$ ). IR (ATR): $v=3083$, 3061, 3034, 2976, 2923, 2865, 1574, 1499, 1454, 1365, 1205, $1067 \mathrm{~cm}^{-1}$. ${ }^{1} \mathrm{H}$ NMR $\left(400 \mathrm{MHz}, \mathrm{CDCl}_{3}\right): \delta=7.38-7.19(\mathrm{~m}, 10 \mathrm{H}), 5.15(\mathrm{q}, J=6.7 \mathrm{~Hz}$, $1 \mathrm{H}), 4.63(\mathrm{~d}, J=11.7 \mathrm{~Hz}, 1 \mathrm{H}), 4.57(\mathrm{~d}, J=11.7 \mathrm{~Hz}, 1 \mathrm{H}), 4.43$ (ddd, $J=$ 14.1, 8.7, 2.3 Hz, $1 \mathrm{H}), 4.09(\mathrm{dd}, J=14.1,7.0,2.0 \mathrm{~Hz}, 1 \mathrm{H}), 3.16-3.06(\mathrm{~m}$, $2 \mathrm{H}), 1.42(\mathrm{~d}, J=6.7 \mathrm{~Hz}, 3 \mathrm{H}), 1.07(\mathrm{~d}, J=6.7 \mathrm{~Hz}, 3 \mathrm{H}) \mathrm{ppm} .{ }^{13} \mathrm{C} \mathrm{NMR}$ 
$\left(100.6 \mathrm{MHz}, \mathrm{CDCl}_{3}\right): \delta=149.9,141.1,138.2,129.2,128.5,127.8,127.8$, 127.6, 127.0, 72.0, 69.0, 68.6, 46.4, 46.4, 18.3, 17.3 ppm. HRMS (ESI) calcd. for $\mathrm{C}_{20} \mathrm{H}_{24} \mathrm{NO}_{2}[\mathrm{M}+\mathrm{H}]^{+}$: 294.1852; found: 294.1861 .

(2R,3S,5S)-5-Benzyloxy-3-methyl-4-oxo-2-phenylhexanal oxime (9): Thiophenol $(140 \mu \mathrm{L}, 1.35 \mathrm{mmol})$ and triethylamine $(210 \mu \mathrm{L}, 1.5 \mathrm{mmol})$ were added to a suspension of $\mathrm{SnCl}_{2}(86 \mathrm{mg}, 0.45 \mathrm{mmol})$ in $\mathrm{CH}_{3} \mathrm{CN}(0.6$ $\mathrm{mL}$ ) at room temperature. Then, a solution of nitroalkane 2a (103 mg, 0.3 $\mathrm{mmol}$ ) in $\mathrm{CH}_{3} \mathrm{CN}(1.2 \mathrm{~mL})$ was added carefully and the resultant mixture was stirred for $30 \mathrm{~min}$. The mixture was concentrated under vacuum and the residue was purified by flash column chromatography (hexanes/EtOAc $80: 20)$ to afford $73 \mathrm{mg}(0.23 \mathrm{mmol}, 75 \%$ yield) of (2R,3S,5S,)-5-benzyloxy-3-methyl-4-oxo-2-phenylhexanal oxime (9) as a yellow oil. $R_{f}$ (hexanes/EtOAc) 0.20. $[\alpha]_{\mathrm{D}}-104.2$ (c 1.3, $\left.\mathrm{CHCl}_{3}\right)$. IR (ATR): $v=3278(\mathrm{br}), 3084,3062,3024,2974,2932,2875,1495,1451,1368$, $1096 \mathrm{~cm}^{-1}$. ${ }^{1} \mathrm{H}$ NMR $\left(400 \mathrm{MHz}, \mathrm{CDCl}_{3}\right): \delta=7.38-7.18(\mathrm{~m}, 11 \mathrm{H}), 4.72(\mathrm{~d}$, $J=11.8 \mathrm{~Hz}, 1 \mathrm{H}), 4.48(\mathrm{~d}, J=11.8 \mathrm{~Hz}, 1 \mathrm{H}), 3.79(\mathrm{q}, J=6.4 \mathrm{~Hz}, 1 \mathrm{H})$, $3.27(\mathrm{dd}, J=11.7,1.5 \mathrm{~Hz}, 1 \mathrm{H}), 2.28(\mathrm{dq}, J=11.7,6.6 \mathrm{~Hz}, 1 \mathrm{H}) 1.40(\mathrm{~d}, J$ $=6.4 \mathrm{~Hz}, 3 \mathrm{H}), 0.83(\mathrm{~d}, J=6.6 \mathrm{~Hz}, 3 \mathrm{H}) \mathrm{ppm} .{ }^{13} \mathrm{C} \mathrm{NMR}(100.6 \mathrm{MHz}$, $\left.\mathrm{CDCl}_{3}\right): \delta=152.1,139.2,138.0,129.1,129.0,128.5,128.0,127.9,127.6$ 98.9, 76.6, 71.4, 43.4, 34.7, 13.0, 12.6 ppm. HRMS (ESI): calcd. for $\mathrm{C}_{20} \mathrm{H}_{24} \mathrm{NO}_{3}[\mathrm{M}+\mathrm{H}]^{+}:$326.1751; found: 326.1750 .

(2R,3S,5S)-5-Benzyloxy-3-methyl-4-oxo-2-phenylhexanenitrile (10): A solution of nitroalkane $2 \mathrm{a}(34 \mathrm{mg}, 0.1 \mathrm{mmol})$ in $\mathrm{CH}_{2} \mathrm{Cl}_{2}(0.5 \mathrm{~mL})$ was added to a stirred suspension of $\mathrm{Sn}(\mathrm{SPh})_{4}(11 \mathrm{mg}, 20 \mu \mathrm{mol})$, DMAP (13 $\mathrm{mg}, 0.11 \mathrm{mmol}), 1 \mathrm{M} \mathrm{Me} 3 \mathrm{P}$ in THF (220 $\mu \mathrm{L}, 0.22 \mathrm{mmol})$, and 40\% DEAD in toluene $(50 \mu \mathrm{L}, 0.11 \mathrm{mmol})$ in $\mathrm{CH}_{2} \mathrm{Cl}_{2}(0.5 \mathrm{~mL})$ at $0^{\circ} \mathrm{C}$ and the resulting mixture was stirred for $30 \mathrm{~min}$ at $0^{\circ} \mathrm{C}$. The, the reaction mixture was concentrated under vacuum and the residue was purified by flash column chromatography (hexanes/EtOAc $90: 10)$ to afford $28 \mathrm{mg}(90 \mu \mathrm{mol}, 93 \%$ yield) of $(2 R, 3 S, 5 S)$-5-benzyloxy-3-methyl-4-oxo-2-phenylhexanenitrile (10) as a colorless oil. $R_{f}$ (hexanes/EtOAc) 0.2. [ $\left.\alpha\right]_{\mathrm{D}}-104.2\left(c 1.3, \mathrm{CHCl}_{3}\right)$. IR (ATR): $v=3084,3059,3031,2974,2929,2869 \mathrm{~cm}^{-1} .{ }^{1} \mathrm{H}$ NMR $(400$ $\left.\mathrm{MHz}, \mathrm{CDCl}_{3}\right): \delta=7.40-7.28(\mathrm{~m}, 10 \mathrm{H}), 4.56(\mathrm{~s}, 2 \mathrm{H}), 4.10(\mathrm{q}, \mathrm{J}=6.9 \mathrm{~Hz}, 1$ $\mathrm{H}), 3.94(\mathrm{~d}, J=9.5 \mathrm{~Hz}, 1 \mathrm{H}), 3.57(\mathrm{dq}, J=9.5,7.0 \mathrm{~Hz}, 1 \mathrm{H}) 1.35(\mathrm{~d}, J=$ $6.9 \mathrm{~Hz}, 3 \mathrm{H}$ ), 1.01 (d, $J=7.0 \mathrm{~Hz}, 3 \mathrm{H}) \mathrm{ppm} .{ }^{13} \mathrm{C} \mathrm{NMR}(100.6 \mathrm{MHz}$, $\left.\mathrm{CDCl}_{3}\right): \delta=212.0,137.5,133.5,129.2,128.7,128.7,128.6,128.1,127.9$ $120.2,80.0,71.7,46.0,39.4,16.4,15.6$ ppm. HRMS (ESI): calcd. for $\mathrm{C}_{20} \mathrm{H}_{25} \mathrm{~N}_{2} \mathrm{O}_{2}\left[\mathrm{M}+\mathrm{NH}_{4}\right]^{+}: 325.1913$; found: 325.1913

\section{Acknowledgements}

Financial support from the Spanish Ministerio de Economía y Competitividad and Fondos Feder (Grant Nos. CTQ2012-31034 and CTQ2015-65759) and the Generalitat de Catalunya (2009 SGR825 and 2014SGR586) as well as a doctorate studentship to A. G.-P. (APIF, Universitat de Barcelona) are acknowledged.

Keywords: Michael addition - Diastereoselectivity • Substrate control $\cdot$ Chiral ketone $\cdot$ Titanium

[1] a) D. A. Oare, C. H. Heathcock in Topics in Stereochemistry, Vol. 19 (Eds.: E. L. Elliel, S. H. Wilen, N. L. Allinger), Wiley, New York, 1989, pp. 207-408; b) B. L. Feringa, J. F. G. A. Jansen in Houben-Weyl. Methods of Organic Chemistry, Vol. E21b (Eds.: G. Helmchen, R. W Hoffmann, J. Mulzer, E. Schaumann), Georg Thieme, Stuttgart, 1995, pp. 2104-2156.

[2] a) T. Hudlicky, J. W. Reed in The Way of Synthesis, Wiley-VCH, Weinheim, 2007; b) E. M. Carreira, L. Kvaerno in Classics in Stereoselective Synthesis, Wiley-VCH, Weinheim, 2009, pp. 389-429.
[3] For reviews on asymmetric Michael reactions, see: a) J. Christoffers, G Koripelly, A. Rosiak, M. Rössle, Synthesis 2007, 1279-1300; b) J. L. Vicario, D. Badía, L. Carrillo, Synthesis 2007, 2065-2092; c) S. Mukherjee, J. W. Yang, S. Hoffmann, B. List, Chem. Rev. 2007, 107, 5471-5569; d) G. P. Howell, Org. Process Res. Dev. 2012, 16, 1258 1272.

[4] a) K. C. Nicolaou, E. J. Sorensen in Classics in Total Synthesis, WileyVCH, Weinheim, 1996; b) K. C. Nicolaou, S. A. Snyder in Classics in Total Synthesis II, Wiley-VCH, Weinheim, 2003; c) K. C. Nicolaou, J. S. Chen in Classics in Total Synthesis III, Wiley-VCH, Weinheim, 2011.

[5] C. Hui, F. Pu, J. Xu, Chem. Eur. J. 2017, 23, 4023-4036.

[6] For representative examples, see: a) T. Arai, H. Sasai, K. Aoe, K. Okamura, T. Date, M. Shibasaki, Angew. Chem. Int. Ed. Engl. 1996, 35, 104-106; b) Y. Hamashima, D. Hotta, M. Sodeoka, J. Am. Chem. Soc. 2002, 124, 11240-11241; c) D. A. Evans, S. Mito, D. Seidel, J. Am. Chem. Soc. 2007, 129, 11583-11592.

[7] For Michael additions of heteroaryl ketones, see: a) A. J. Simpson, H. W. Lam, Org. Lett. 2013, 15, 2586-2589; b) D. Yang, L. Wang, D. Li, F. Han, D. Zhao, R. Wang, Chem. Eur. J. 2015, 21, 1458-1462; c) D. Yang, D. Li, L. Wang, D. Zhao, R. Wang, J. Org. Chem. 2015, 80, 4336-4348.

[8] For highly enantioselective direct-type conjugate additions of less acidic amides, see: a) H. Suzuki, I. Sato, Y. Yamashita, S. Kobayashi, J. Am. Chem. Soc. 2015, 137, 4336-4339; b) N. Majumdar, A. Saito, L. Yin, N. Kumagai, M. Shibasaki, Org. Lett. 2015, 17, 3362-3365.

[9] For representative examples, see: a) D. A. Evans, M. T. Bilodeau, T. C. Somers, J. Clardy, D. Cherry, Y. Kato, J. Org. Chem. 1991, 56, 57505752; b) J. Mulzer, R. Zuhse, R. Schmiechen, Angew. Chem. Int. Ed. Engl. 1992, 31, 870-872; c) T. Hintermann, D. Seebach, Helv. Chim. Acta 1998, 81, 2093-2126; d) A. Job, C. F. Janeck, W. Bettray, R. Peters, D. Enders, Tetrahedron 2002, 58, 2253-2329; e) J. H. Smitrovich, L. DiMichele, C. Qu, G. N. Boice, T. D. Nelson, M. A. Huffman, J. Murry, J. Org. Chem. 2004, 69, 1903-1908; f) A. Olivella, C. Rodríguez-Escrich, F. Urpí, J. Vilarrasa, J. Org. Chem. 2008, 73, 1578-1581; g) P. Lu, J. J. Jackson, J. A. Eickhoff, A. Zakarian, J. Am. Chem. Soc. 2015, 137, 656-659.

[10] a) C. Palomo, J. M. Aizpurua, M. Oiarbide, J. M. García, A. González, I. Odriozola, A. Linden, Tetrahedron Lett. 2001, 42, 4829-4831; b) N. Germain, D. Schlaefli, M. Chellat, S. Rosset, A. Alexakis, Org. Lett. 2014, 16, 2006-2009; c) A. A. S. Gietter-Burch, R. E. Mitrut, D. A. Watson, Org. Lett. 2015, 17, 5468-5471.

[11] For the influence of chiral enones as Michael acceptors in double asymmetric organocatalytic conjugate additions, see I. Iriarte, S. Vera, E. Badiola, A. Mielgo, M. Oiarbide, J. M. García, J. M. Odriozola, C. Palomo, Chem. Eur. J. 2016, 22, 13690-13696.

[12] a) J. G. Solsona, P. Romea, F. Urpí, J. Vilarrasa, Org. Lett. 2003, 5, 519-522; b) J. G. Solsona, P. Romea, F. Urpí, Tetrahedron Lett. 2004, 45, 5379-5382; c) M. Pellicena, J. G. Solsona, P. Romea, F. Urpí, Tetrahedron 2012, 68, 10338-10350; d) S. Alcoberro, A. GómezPalomino, R. Solà, P. Romea, F. Urpí, M. Font-Bardia, Org. Lett. 2014, 16, 584-587.

[13] M. Fàbregas, A. Gómez-Palomino, M. Pellicena, Daniel F. Reina, P. Romea, F. Urpí, M. Font-Bardia, Org. Lett. 2014, 16, 6220-6223.

[14] For a review on asymmetric Michael additions to nitroalkenes, see O. M. Berner, L. Tedeschi, D. Enders, Eur. J. Org. Chem. 2002, 1877-1894.

[15] M. Ferreró, M. Galobardes, R. Martín, T. Montes, P. Romea, R. Rovira, F. Urpí, J. Vilarrasa, Synthesis 2000, 1608-1614.

[16] M. Brenner, D. Seebach, Helv. Chim. Acta 1999, 82, 2365-2379.

[17] Nitroalkenes $\mathbf{a}-\mathbf{g}$ were prepared according to standard pprocedures, see D. E. Worrall, Org. Synth. 1929, 9, 66-67.

[18] For the preparation of nitroalkenes $\mathbf{h}-\mathbf{m}$ see reference $7 \mathbf{a}$.

[19] Crystallographic data for $\mathbf{2} \mathbf{g}$ and $\mathbf{3 a}$ have been deposited at the Cambridge Crystallographic Data Centre as supplementary publication no. CCDC 1560404 and 1560403 respectively. These data can be 
obtained free of charge from The Cambridge Crystallographic Data Centre via www.ccdc.cam.ac.uk/data_request/cif.

[20] For the preparation of ketones $\mathbf{4}$ and $\mathbf{5}$, see reference 15.

[21] For an early proposal for the participation of such a transoid species, see reference 16 .

[22] G. Deng, X. Tian, Z. Qu, J. Wang, Angew. Chem. Int. Ed. 2002, 41, 2773-2775.

[23] Computational studies on intermolecular Michael additions of lithium enolates to $\alpha, \beta$-unsaturated esters indicate that these reactions proceed through eight membered cyclic transition states, see E. E. Kwan, D. A. Evans, Org. Lett. 2010, 12, 5124-5127.

[24] J. O. Osby, B. Ganem, Tetrahedron Lett. 1985, 26, 6413-6416.

[25] M. Bartra, P. Romea, F. Urpí, J. Vilarrasa, Tetrahedron 1990, 46, 587594.

[26] F. Urpí, J. Vilarrasa, Tetrahedron Lett. 1990, 31, 7497-7498. 


\section{Entry for the Table of Contents}

\section{FULL PAPER}

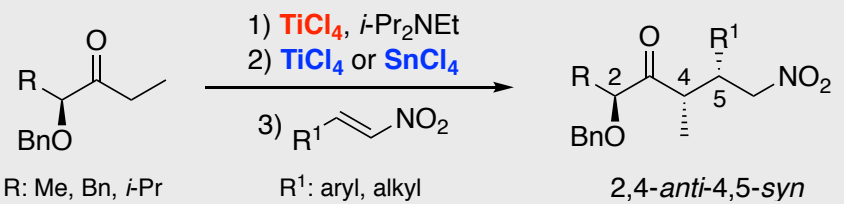

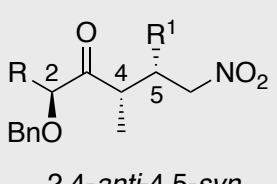

Lewis acid-mediated Michael additions of titanium enolates of chiral $\alpha$-benzyloxy ketones to conjugated nitroalkenes proceed with high diastereoselectivity in good yields
Stereoselective Michael additions

Alejandro Gómez-Palomino, Adrián Barrios, Pedro García-Lorente, Pedro Romea, ${ }^{*}$ Fèlix Urpi ${ }^{*}$ and Mercè FontBardia

Page No. - Page No.

Substrate-Controlled Michael Additions of Titanium Enolates from Chiral $\alpha$-Benzyloxy Ketones to Conjugated Nitroalkenes 